\title{
Self Noise and Contrast Controlled Thinning of gray image
}

\author{
Rabaa Youssef ${ }^{\mathrm{a}, 1, *}$, Sylvie Sevestre-Ghalila ${ }^{\mathrm{b}, * *}$, Anne Ricordeau ${ }^{\mathrm{c}}$, Amel Benazza $^{\mathrm{a}}$ \\ ${ }^{a}$ COSIM Lab. of Sup'Com. Technological City of Communications, Raoued, 2083 Ariana, Tunisia \\ ${ }^{b}$ CEA-LinkLab in Telnet Innovation Labs. Technological City of Communications, Raoued, 2083 Ariana, Tunisia \\ ${ }^{c}$ MAP5 Lab. of Paris Descartes. 45 rue des Saints Pères, 75270 Paris Cedex 06
}

\begin{abstract}
Homotopic grayscale thinning leads to "over-connected skeleton" when applied on noisy images. One way to avoid this phenomenon is the parametric thinning. It consists in relaxing the initial constraint by lowering low contrast crests, peaks and ends, according to a manually selected parameter and under the constraint of ascendant gray level processing. We propose to control this parameter by considering the lowering decision in a statistical framework of hypothesis test under the assumption of an additive Gaussian noise. A unitary hypothesis test based on the minimum test statistic is used for the elimination of peaks and noise related extremities, while a fusion of multiple tests is required for the insignificant crest lowering decision. This leads to a local adjustment and a standardization of the parametric thinning process that depends only on the chosen significance level of the test.
\end{abstract}

Keywords: Thinning, homotopy, hypothesis test, fusion, evaluation

\section{Introduction}

Skeletonization is an image transformation that aims to represent objects by their medial axis lines while preserving image topology. It results in a one-pixel thin line called skeleton. The main approaches of skeletonization are based either on differential geometry or mathematical morphology.

The differential methods category is widely used in road extraction from aerial images applications. A first example of such methods is the line detector of [1]. It models the gray objects as a surface and uses the direction maximizing the concave curvature of gradient image to localize ridge points. As a result of differential geometry, this direction corresponds to the eigenvector associated to the minimum negative eigenvalue of the Hessian matrix $H_{S}$. Other methods that combine differential geometry with region-context 2] or with snakes edge detection method [3] offer multi-resolution approaches. Despite the accuracy of these medial axis detectors, their complex parameters adjustments and especially their

\footnotetext{
*Principal corresponding author

** Corresponding author

Email addresses: rabaa.youssef@supcom.tn (Rabaa Youssef), sylvie.ghalila@cea.fr (Sylvie Sevestre-Ghalila), a.ricordeau@iut.univ-paris8.fr (Anne Ricordeau), benazza.amel@supcom.rnu.tn (Amel Benazza)

${ }^{1} \mathrm{Tel}:(+216) 98930967$
} 
inability to ensure the connectedness of objects (homotopy of skeleton) orients their applicability to regular object lines with few junctions (like roads, vessels etc). Concerning the morphology based skeletonization category, thinning methodologies are of primary interest. In fact, homotopic thinning of binary images has been widely investigated in the early 90's by L. Lam [4], B-K Jang [5, 6], pointing out their role in feature extraction as an important step of pattern recognition applications [7, 8, 9]. Moreover, interest in homotopic thinning has reached other applications such as road extraction [10, 11], medical field [12] and biometrics [13, 14].

The binary homotopic thinning methods are either parallel or sequential. A review of parallel approaches has been proposed by [15]. As to sequential approaches, they proceed by iterative removal of simple pixels that does not change topological characteristics and preserves unattached branches from deletion via blocking endpoints as explained by A. Rosenfeld in [16].

In this work, we are interested in gray tone images where no formal separation of the foreground and background subsets can be assumed. Concerning parallel thinning approaches, few were proposed in the grayscale context [17, 18], while a larger interest to sequential ones was shown in the litterature for the framework extensions it allows. For instance, one of the possible extensions was proposed by Arcelli and Serino in [19]. The authors decompose the image into different subsets having homogeneous gray values via a labelling step. The labelling aims to guide the thinning operations to the regions of the foreground by first proceeding low gray-valued subsets. This technique enables the use of binary thinning notions on each labelled subset. However, it requires both a preprocessing and a postprocessing in order to obtain a regular skeleton. Another possible way to extend the use of binary notions to the grayscale context was investigated by J. Serra [20], S. Beucher [21] who proposed a cross-sectional processing of the image gray levels. In fact, the cross-sectional topology allows to state that a pixel is simple for the binary cross-section composed of higher gray values of the 8-neighborhood and thus, simple for the gray tone image. This extension was formalized by G. Bertrand [22] and M. Couprie [23] who fully described the first gray thinning algorithm. The authors also defined endpoint pixel in the grayscale case in order to impede the lowering of branche's extremity, as performed by A. Rosenfeld [16] in the binary case.

Regarding this new topological gray thinning framework, homotopy is ensured by lowering gray values during the peeling step. After peeling the topographic relief until stability, the expected gray skeleton is a gray image composed of regional minima and high-valued pixels that can be considered as "crest pixels".

In addition to the topology preservation, consideration of noisy pixels is an important improvement 45 of the thinning framework. In fact, the sequential gray thinning method might maintain insignificant crests, extremities and isolated pixels and results in "over-connected" and inexploitable skeletons. 
In a recent work, Couprie et al. proposed a gray parallel thinning method [24]. The authors use critical kernels, first introduced by Bertrand [25], in order to propose a parallel thinning strategy guaranteeing topology preservation. The originality of this method lies in its applicability to the 3D domain thanks to the use of the cubical complex framework. However, its sensitivity to the presence of noise produces insignificant crest lines that are post-processed according to their level of contrast.

To avoid pre-processing and post-processing steps that may result in a loss of information, Couprie et al. 23] previously introduced a gray thinning that relaxes the strong constraint of preserving connectedness. The authors relax this constraint by merging the simple pixel definition with new ones to eliminate insignificant peaks, crests and endpoints. In fact, this algorithm defines topological notions describing the noise-related pixels, examines first pixels of minimal gray level and adjusts the lowering condition using a parameter denoted $\lambda$. Therefore, thinning insignificant branches from the skeleton requires an accurate setting of the lowering parameter $\lambda$.

60

A standardized adjustment of this parameter appears more suitable for a convenient use of the method. In a first attempt, we proved empirically the existing link between this parameter and the level of noise on images. By means of subjective tests on skeleton quality, we established the linear dependency of the contrast parameter to the standard deviation of noise [26]. Second, we deduced that the lowering step in the thinning requires a decision that could be assimilated to a statistical hypothesis test. Therefore, a simple statistical test based on the range statistic was defined in [27] for the parametric thinning in order to choose the contrast parameter at the upper bound of the confidence interval. This chosen value allows to eliminate most of the noisy pixels and limits the lowering error rate. This error control is fixed by well-known significance test levels. It consists of an acceptable error rate of classification (lowerable vs. not lowerable) and stands for a measure that has more sense for common users of the thinning method.

It was noted, at the end of the initial work [27], that the statistical test which involves the insignificant crests is actually, the fusion of multiple tests hypothesis. Therefore, more accurate global hypothesis decomposition needs to be defined in order to better choose confidence level for each individual hypothesis.

In the present work, we first revisit the lowering criterion for peak and endpoint configurations in order to eliminate more efficiently noisy ones. Second, we use the statistical hypothesis test framework to locally adjust the thinning parameter for insignificant peak and endpoint. By building a test based on the lowering criterion, we show that the thinning parameter is no longer global but needs to be set locally as a function of the size and number of connected components in the low neighborhood of each 
pixel. The local thinning parameter values can thus be precalculated at fixed test significance levels and for known noise standard deviation. This unitary test is then used in the fusion of multiple tests required for crest configurations. The global significance level for these configurations is a function of unitary test levels that are calculated as a function of the number of connected components (i.e tests). Therefore, the proposed adjustment of the thinning is based on the noise standard deviation and on the chosen error decision which reflects the contrast preservation level. We refer to the proposed method as Self Contrast Controlled Thinning (SCCT).

In the first section, we present the evolution of the thinning framework, from binary to classic gray level algorithm. In the second section, we focus on the new lowering criterion for peak and end pixels and then on the proposed statistical control of the parametric thinning. We conclude the section by presenting the challenges in terms of algorithmic optimization and topology preservation of the implemented solution. The third section is dedicated firstly to a quantitative evaluation of (SCCT), compared to the skeletonization method $\lambda$-skeleton available in Pink library and to the Differential Line Detector (DLD) of [1]. Secondly, an illustration on real images from applications of interest such as biomedical imaging, biometrics and character recognition is given.

\section{Topological framework of the thinning: from binary to parametric gray thinning}

Multiple binary skeletonization approaches, in particular thinning methods, were proposed in the 90's. Their respective performances were quantitatively evaluated [4] [5] according to criteria linked to skeleton connectedness, geometry preservation and execution times. Applying these methods on grayscale images is possible only through preprocessing and binarization techniques. However, these preprocessing steps might generate a loss of information that degrades the quality and relevance of the obtained skeletons. In order to better control this loss of information, specific thinning methodologies were proposed for grayscale images. As mentioned in the introduction, the most popular approach to extend binary thinning notions to gray image context exploit the cross-sectional topology. In addition, new solutions for the noise information removal were proposed such as the parametric thinning introduced in [23].

The understanding of the parametric gray thinning procedure, which is the starting point for our work, needs the definition of topological prerequisites. Describing such a framework starts naturally from the basic notions of the binary case and continues with the gray thinning framework. We choose to follow this evolution and detail, in Subsection 1.1, the binary thinning principle and its topological definitions. We explain in Subsection 1.2 the topological thinning extension from binary to grayscale domain and detail the parametric thinning algorithm that filters noisy information. 


\subsection{Binary thinning}

A binary image consists of background pixels and foreground pixels also named object pixels. Let us denote this value for the pixel $x$ by $I(x) \in\{0,1\}$ where $\{x, I(x)=1\}$ refers to object pixels and $\{x, I(x)=0\}$ refers to the background pixels. Applied to a binary image, the goal of homotopic thinning is to remove object pixels without changing the topological characteristics of the image. Since complementary connectivity between background and foreground must be chosen to respect the Jordan Curve Theorem, 4-connectivity is classically used for the background and 8connectivity for the foreground.

We denote by $\mathcal{N}_{4}(x)$ the set of 4 -nearest neighbors of a pixel $x$ and by $\mathcal{N}_{8}(x)$ the set of its 8 -nearest neighbors.

Thinning deletable pixels are called simple pixels in [16] 28] and are defined as follows:

Definition 1. A simple pixel $x$ verifies the following two conditions:

(i) $\mathcal{N}_{4}^{0}(x)=\left\{y \in \mathcal{N}_{4}(x), I(y)=0\right\} \neq \emptyset$,

(ii) $\mathcal{N}_{8}^{1}(x)$ is 8-connected and unique, where $\mathcal{N}_{8}^{1}(x)=\left\{y \in \mathcal{N}_{8}(x), I(y)=1\right\}$.

The condition (i) states simply that $x$ is a border point and condition (ii) that removing $x$ doesn't change the object connectedness. The binary thinning proceeds, by first detecting the simple pixels set and second, deleting iteratively these pixels. The sequential binary thinning procedure is given by the algorithm 1, A binary thinning procedure only based on simple pixel notion leads to an ultimate skeleton where no branches are preserved since no condition on extremities is stated. This is useful for closed objects with no extremities to preserve such as the rings image of Figure 2](a) that leads to a skeleton (b) with no extremities. A thinning that is conditioned by the constraint "simple but not endpoint" as explained in Algorithm 1, leads to a skeleton that preserves the geometry of objects in addition to the topology preservation. This thinning criterion is used for objects that present free extremities as illustrated by Figure 2,(c) and (d). Isolated peaks can also be detected and removed from the skeletonized image if we add to the selection list $\mathcal{L}$ of Algorithm 1 the statement including peaks in the deletion step. 


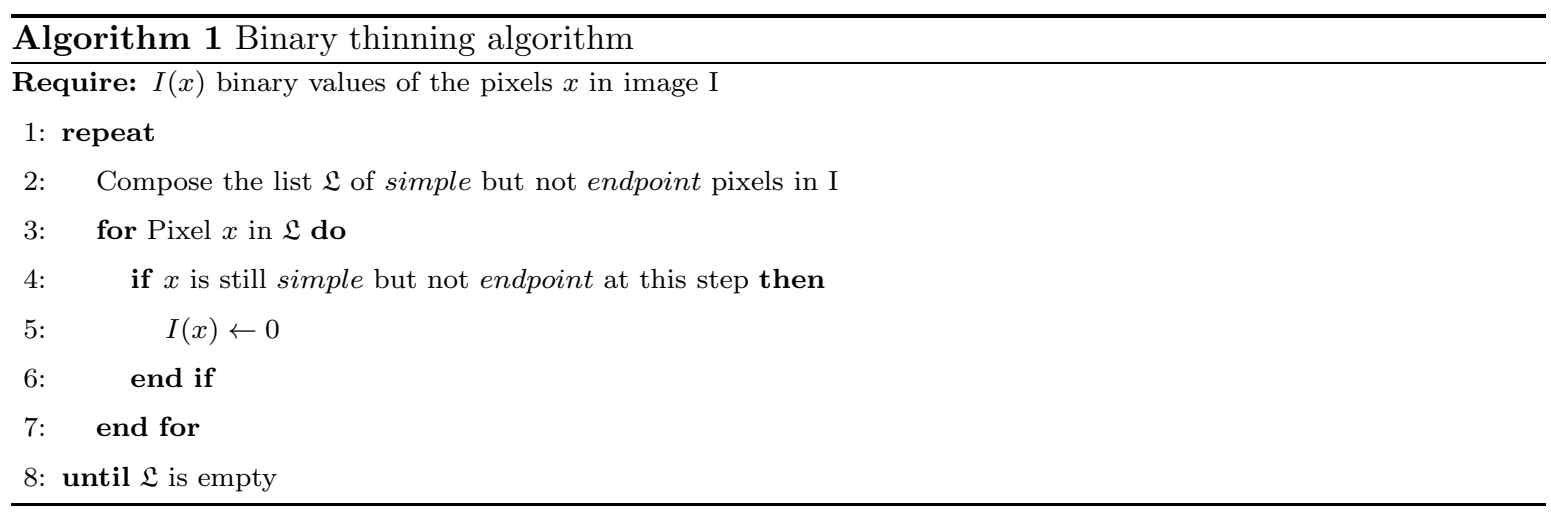

Definition 2. An object pixel $x$ is an endpoint if it has only one 8-neighbor belonging to the foreground: $\left|\mathcal{N}_{8}^{1}(x)\right|=1$.

An object pixel $x$ is a peak if it has no 8-neighbor belonging to the foreground: $\left|\mathcal{N}_{8}^{1}(x)\right|=0$.

Figure 1 shows examples of the three binary notions of simple, peak and endpoint.

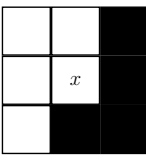

(a)

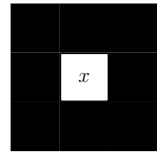

(b)

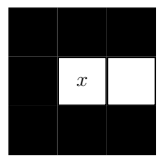

(c)

Figure 1: Topological configurations for central pixel $x$. (a): simple, (b): peak, (c): endpoint.

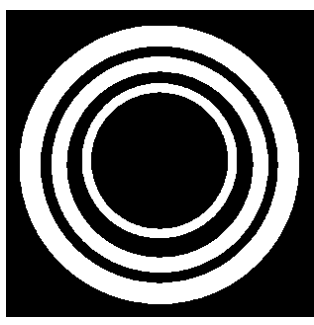

(a)

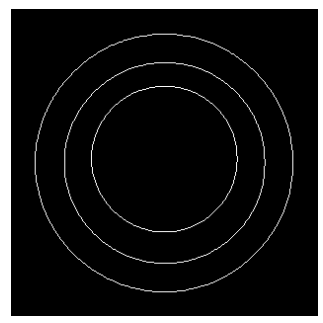

(b)

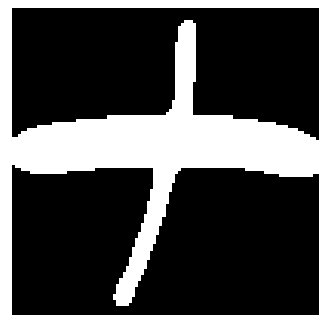

(c)

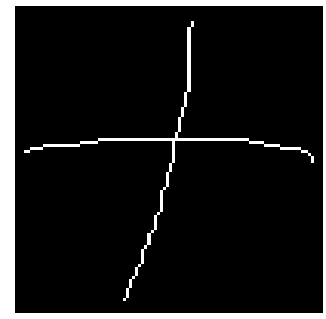

(d)

Figure 2: Binary thinning cases: (a): Rings image $311 \times 311$. (b): Thinning result for (a). (c): Intersection image $100 \times 100$. (d): Thinning of (c) with endpoint preservation.

We note that for binary thinning conditions, the peeling proceeds while preserving the topological characteristics of the object. It results in an homotopic skeleton that visually well-summarizes the geometry of objects with regular edges. In the case where 
a binary object is distorted at the contour, insignificant branches may appear on its final skeleton. In Subsection 1.2, we present the topological framework of conventional gray level thinning and subsequently the Subsection 1.3 is devoted to the parametric thinning [23] that manages the elimination of noisy information.

\subsection{Cross-sectional topology: from binary to gray thinning}

When considering the grayscale case, the notion of borders is no longer obvious, unless we proceed by binarizing the image, which may lead to a loss of information. A grayscale image can be seen as a topographic relief where the gray value of a pixel corresponds to its height on the relief. Let us define this value for the pixel $x$ as $I(x) \in\left\{0,1, . ., I_{\max }\right\}$. The extension of the thinning algorithm to the grayscale domain is based on the definition of the cross-sectional topology introduced in [20]. The cross-section of an image $I$ at level $l$ refers to the binary image $I_{l}=\{y, I(y) \geq l\}$. For a gray tone image, J. Serra [20] stated that a pixel $x$ is simple for the gray image $I$ if it is simple for the binary cross section $I_{l}$ where $l=I(x)$.

An extension to the grayscale case of peak and endpoint definitions was also made using the crosssectional topology. Figure 3 shows a configuration where the central pixel $x$ is respectively identified as simple, peak and endpoint according to the cross-section $I_{l}(x)$ composed of $x$ and its upper neighbors $N_{8}^{>}(x)=\left\{y \in N_{8}(x), I(y) \geq I(x)\right\}$.

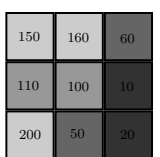

(a)

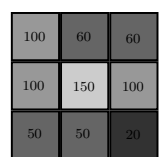

(b)

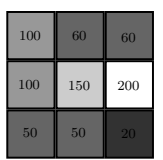

(b)

Figure 3: (a): central pixel is simple. (b):central pixel is peak. (c):central pixel is endpoint.

This naive extension of the thinning to gray tone image has the same algorithmic structure as the binary one. According to the binary algorithm 1 , after constructing the list of simple pixels, the second phase of the thinning in gray level case should be the removal of these pixels. However, in the gray case, the binary deletion of border pixels is equivalent to the lowering of pixels intensity. Repeated unitary lowerings of the gray value of a simple pixel remain homotopic transformations until reaching the greatest value of darker 8-neighbors. Therefore, the choice of the new intensity level could be set directly to:

$$
\max \left\{I(y), y \in \mathcal{N}_{8}^{<}(x)\right\}, \text { where } \mathcal{N}_{8}^{<}(x)=\left\{y \in \mathcal{N}_{8}(x), I(y)<I(x)\right\}
$$
are positioned on the highest lines of the relief after iteratively lowering gray levels from the darkest to 
the brightest ones (simple pixels are naturally positioned at darker lines in first iterations). This result is illustrated by the initial gray image of Figure 4.(a) and its superposed skeleton in red. However, for a noisy image, the basic gray level thinning method leads to "over-connected skeleton", where insignificant loops, branches and extremities related to the presence of noise appear. For example, when applying an additive white Gaussian noise to the synthetic image of Figure 4.(b), the result of the basic gray thinning operation of Figure 4 (c) shows the emergence of low contrasted crests, peaks and extremities on the background. These new elements cannot be lowered by the thinning process, since their elimination implies a change of the initial cross-section topology. In fact, the homotopic thinning criterion does not allow any change of the topology and does not dissociate noise related information from its significant part. The profile image (d) of Figure 4 shows the displacement of the bold red skeleton profile compared to the ideal one (in red and dashed), in addition to the emergence of low peaks on this profile that corresponds to insignificant crests.

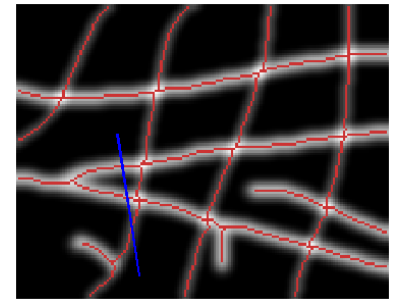

(a)

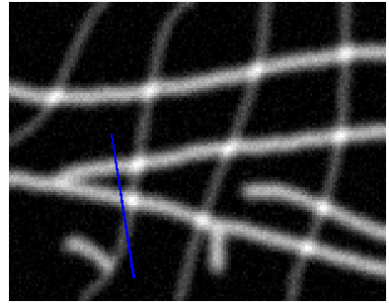

(b)

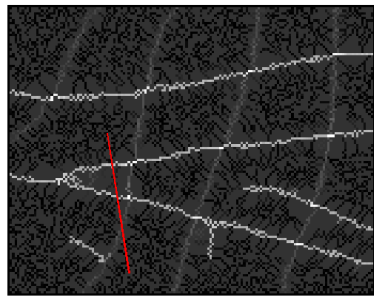

(c)

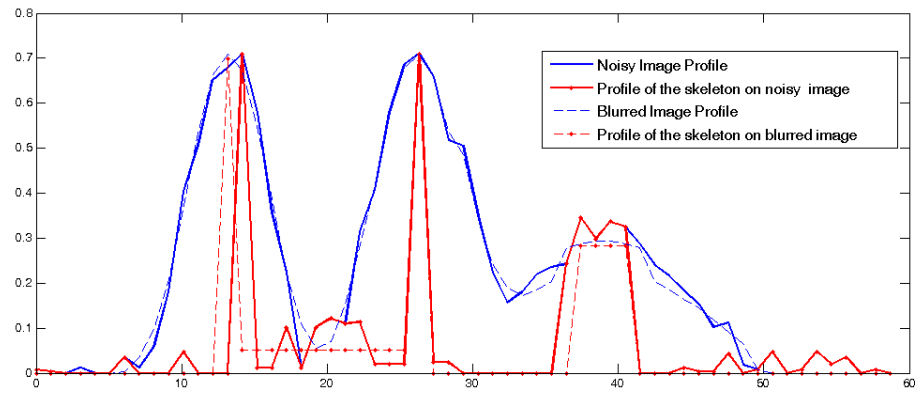

(d)

Figure 4: Basic thinning algorithm applied on synthetic image $153 \times 121$ gray values in [0..1]. (a): Skeleton in red above ideal gray relief. The binary skeleton is obtained by detecting regional minima of the gray skeleton image. (b): Noisy image obtained by adding white Gaussian noise to (a) $\sigma=3 \%$. (c): Gray skeleton of (b) using brighter background gray level to distinguish low-contrasted crests. (d): Profile of ideal image (a) and its skeleton in dashed lines. Profile of noisy image (b) and its skeleton (c) in bold.

The extension from binary to gray level case allowed the thinning to directly operate on the gray levels of the image, with no pre-processing or binarization. However, this extension has difficulties 
in managing noise related information as illustrated by example of Figure 4. In Subsection 1.3, we present a more flexible version of the thinning that relaxes the strong constraint of homotopy in order to eliminate insignificant information.

\subsection{Parametric thinning framework and implementation}

The thinning procedure needs to detect only significant skeleton information. Due to the strong constraint of topology preservation imposed by the use of simple notion only, managing noise elimination is not possible since the basic thinning does not differentiate pixels configurations by their significance. The parametric thinning proposed in [23] permits the lowering of not only simple pixels, but also low-contrasted pixels as peaks, crests and endpoints.

\subsubsection{Parametric thinning framework}

The general idea of the method is that thinning constraints can be relaxed when a local contrast is below a global parameter $\lambda$. Related to this idea, definitions of $\lambda$-crest, $\lambda$-end and $\lambda$-peak are detailed in the following.

Definition 3. Pixel $x$ is $\lambda$-crest if it fulfills the two following conditions:

(i) At least 2 connected components $(\mathcal{K} \geq 2)$ in $\mathcal{N}_{8}^{<}(x)$ are 4 -connected and 4-adjacent to $x$;

(ii) For these $\mathcal{K}$ connected components, named $\mathcal{C}_{k}(k=1, . ., \mathcal{K})$, at least $(\mathcal{K}-1)$ are at minor distance from $I(x)$ :

$$
d_{1}\left(x, \mathcal{C}_{k}\right)=\left(I(x)-\min \left\{I\left(\mathcal{C}_{k}\right)\right\}\right) \leq \lambda .
$$

Condition $(i)$ in the $\lambda$-crest Definition 3 , states that pixel $x$ lies locally on a crest surrounded by $\mathcal{K}$ darker components. Condition (ii) states that no more than one darker component is at a distance exceeding $\lambda$, thus that the crest pixel may be assimilated to the local background represented by the other $(\mathcal{K}-1)$ dark components. It is worth noting that 4 -connectedness and 4 -adjacency conditions limits $\mathcal{K}$ to $2 \leq \mathcal{K} \leq 4$, as illustrated in Figure 5 ,
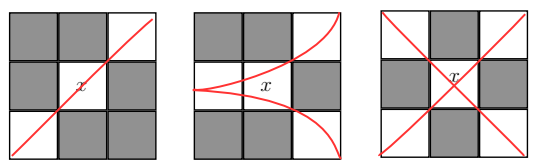

Figure 5: In each one of the configurations, $x$ is $\lambda$-crest for $\mathcal{K}=2$ (left), $\mathcal{K}=3$ (middle) and $\mathcal{K}=4$ (right).

Definition 4. Pixel $x$ is $\lambda$-end (resp. $\lambda$-peak) if $x$ is an end (resp. peak) pixel and:

$$
d_{2}(x, \mathcal{C})=(I(x)-\max \{I(\mathcal{C})\}) \leq \lambda
$$

where $\mathcal{C}$ is the unique 8-connected component of the lower 8-neighborhood of the pixel $x \mathcal{N}_{8}^{<}(x)$. 
These $\lambda$-dependant configurations are merged in the single $\lambda$-lowerable notion of definition 5 .

Definition 5. Pixel $x$ is $\lambda$-lowerable if it satisfies one of the following conditions:

(ii) $x$ is $\lambda$-end

An overall graph of lowering cases for the $\lambda$-thinning is presented in Figure 6, where we differentiate $\lambda$-dependant configurations ( $\lambda$-lowerable) from topology preserving simple pixels (lowerable).

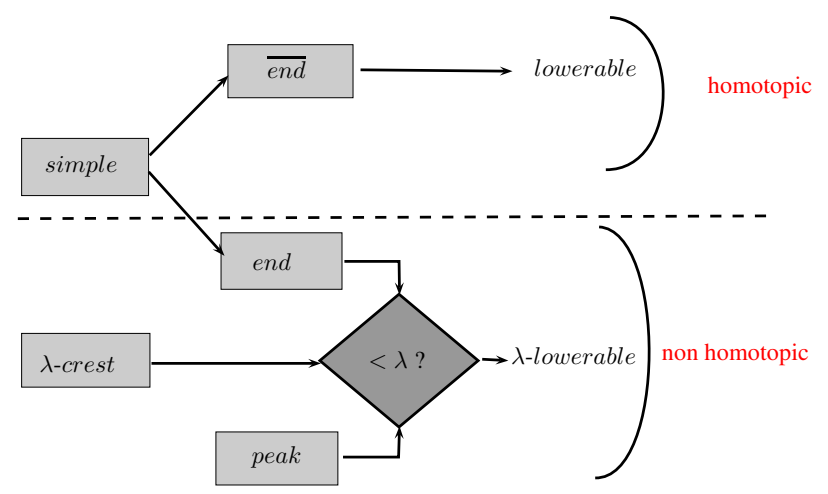

Figure 6: Description of $\lambda$-lowerable pixel.

In Algorithm 2, $\lambda$-lowerable pixels are identified in addition to lowerable pixels. The $\lambda$-lowerable configurations are composed of noise related crests, peaks and ends and are eliminated in a gray priority order during the thinning process as mentioned in line 4 of Algorithm 2, An arbitrary lowering with no ordered gray level treatment may "trespass" the object crest lines and disconnect them. In fact, low crests are located not only in the background, but also in the foreground. Their processing in the first iterations may reduce their local contrast to neighbors and confuse them with the insignificant crests in the upcoming iterations. Therefore, a random lowering with no given priority to gray levels results in disconnecting the object. A random treatment of $\lambda$-lowerable pixels is illustrated by Figure 7. where pixels $b$ and $g$ should be the final skeleton pixels. However, $b$ is seen as $\lambda$-crest because of the unordered gray level treatment. In the appendix of [23], the authors explain that a sorting step on the list of $\lambda$-lowerable pixels needs to be performed before thinning so that object crest disconnections are avoided as much as possible. 

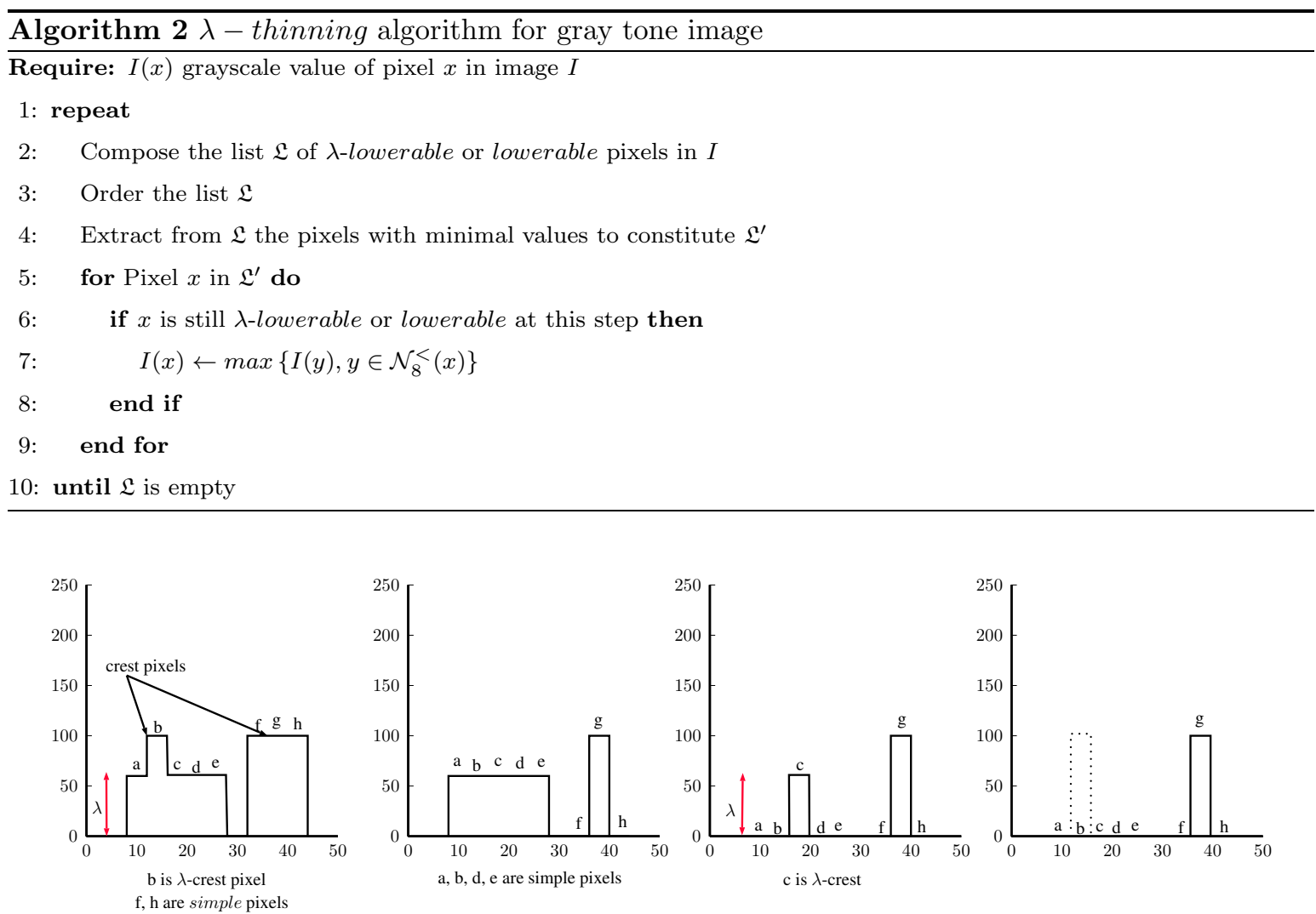

Figure 7: Description of unordered treatment during the $\lambda$-thinning iterations. Profile image is used as per [23].

Two main changes are noted between the classic gray thinning and the parametric thinning of Algorithm 2, The first is the use of homotopy changing configurations in the lowering process so that noisy pixels are filtered. The second is the ascendant treatment of the image gray levels to limit object disconnection induced by the treatment of non-homotopic configurations.

\subsubsection{Implementation of the parametric thinning}

Implementation of Algorithm 2 is available in the Pink library 2 and denoted " $\lambda$-Skeleton". Pink is an image processing toolbox implementing operators of mathematical morphology and other image processing operations, including the $\lambda$-Skeleton method implemented using Hierarchical Queues (HQs) in order to quicken the parametric thinning process as will be explained in Section 2.3. Pink library offers options that permit to specify whether to involve or not the peak and $\lambda$-end criteria in the lowering decision. In fact, in Pink implementation of $\lambda$-Skeleton algorithm, the authors choose to ignore the definition of $\lambda$-peak pixels and to simply eliminate all isolated pixels to improve the results.

${ }^{2}$ http://www.esiee.fr/ coupriem/Pink/doc/html/ 
The lowering of $\lambda$-ends is activated for applications that require the preservation of ends. Figure 8 illustrates implementation of the $\lambda$-Skeleton with different options. According to our experiments, low crest pixels of Figure 4 are completely eliminated, and the initial object connectedness is preserved.

However, the skeleton is more accurate when neglecting all peaks and end points. This result shows the low effectiveness of $\lambda$-end and $\lambda$-peak definitions in lowering unwanted pixels as defined in [23]. These results will be discussed in Subsection 2.1 and new definitions of $\lambda$-end and $\lambda$-peak pixels will be consequently proposed.

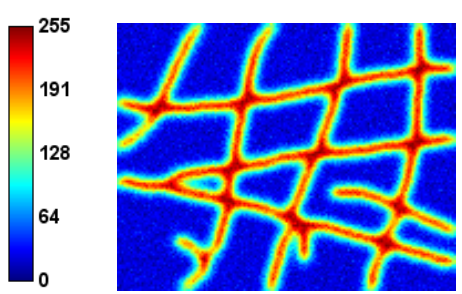

a: Network image

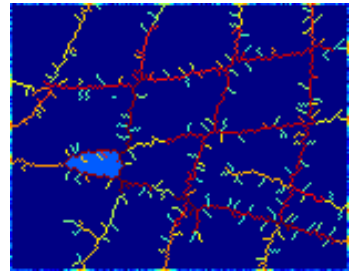

b: Remove peaks and keep high ends

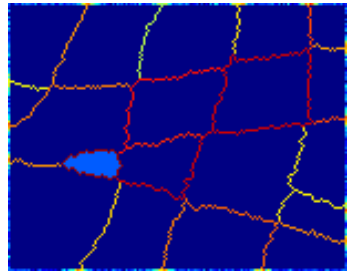

c: Remove ends and peaks

Figure 8: Synthetic network image of figure 4 with additive white Gaussian noise $\sigma=5 . \lambda=25$.

If we assume that the parameter $\lambda$ is set to the adequate value, the parametric thinning results in a thin skeleton that is concordant with the expectations of an expert user. Nevertheless, the manual setting of $\lambda$ remains sensitive to the user expertise and decision. Indeed, the implementation of the parametric thinning with a small valued $\lambda$, results in an "over-connected skeleton". However, an over-estimated parameter induces disconnections especially for low-contrasted branches. Figure 9 shows the effect of $\lambda$ parameter on $\lambda$-Skeleton results. It allows to lower noise-related pixels and at certain values $(\lambda=70)$, it starts disconnecting crests of low-contrasted crests before disconnecting high-contrasted ones. We notice that these low but significant crest lines are the first to be affected by disconnections when over evaluating $\lambda$. These results suggest that the choice of the thinning parameter should depend not only on the image noise but also on the contrast of its objects.

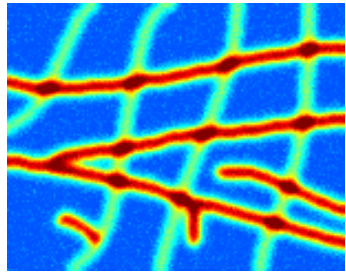

Varying branches contrast

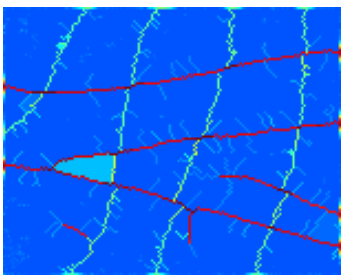

$\lambda=10$

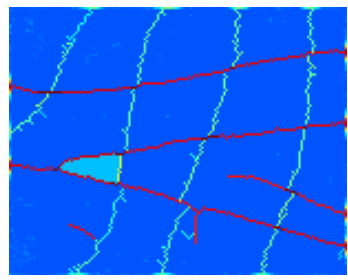

$\lambda=30$

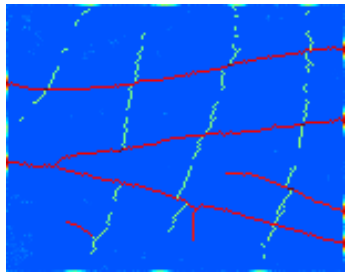

$\lambda=70$

Figure 9: Parametric thinning using different values of $\lambda$ on synthetic noisy image of figure $4 \sigma_{n o i s e}=5$

The parametric thinning gave rise to new challenges for the thinning process: finding the tradeoff 

i.e when the background gray values are more distant from the examined pixels. 


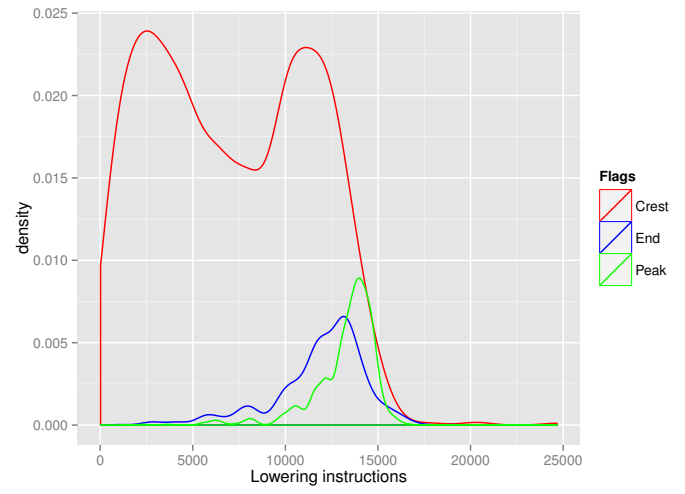

Figure 10: Order treatment of $\lambda$-dependent configurations through successive lowering instructions.

This solution seems to be not sufficiently efficient, since some of the insignificant peaks and extremities still remain on the final skeleton image as shown in Figure 8 of subsection 1.3.2,

Since we choose not to change the treatment order of the parametric thinning, we have to assume the change of the image dynamic during the thinning process. Consequently, we propose to evaluate $\lambda$-peak and $\lambda$-end distance of Definition 4 using the initial image $I_{0}$ gray levels. In fact, each time an end or peak point is detected on the current image, we take into account the initial gray levels to decide whether its height is significant or not. The distance being measured on the original image $I_{0}$, we overcome the dynamic change issue for the upcoming peelings. Finally, we propose to unify this criterion for all $\lambda$-dependent configurations by using a distance inspired from the $\lambda$-crest Definition 3 ,

Definition 6. Pixel $x$ is a $\lambda$-peak (resp. $\lambda$-end) if $x$ is a peak (resp. end) according to the cross section $I_{l}(x)$ and:

$$
d(x, \mathcal{C})=I_{0}(x)-\min \left(I_{0}(C)\right) \leq \lambda
$$

where $\mathcal{C}$ is the unique 8-connected component in $\mathcal{N}_{8}^{<}(x)$ obtained from the current image $I$ and $I_{0}(\mathcal{C})$ is composed of initial gray values of $\mathcal{C}$.

Deciding whether a peak pixel is lowerable or not suggests that the gray level of the considered pixel $x$ should be assimilated to its lower neighbors gray levels of $\mathcal{N}_{8}^{<}(x)$. For example, if the central pixel in Figure 3. (b) is considered as a lowerable peak, then its gray value 150 is closer to the background gray levels $(20,50,60,100)$ than to the object ones. In this case, the lowering requires a parameter value $\lambda \geq 130$ and implies that peaks at smaller height than $d=130$ are insignificant regarding the gray levels of the skeleton we are looking for. This choice arises the issue of setting the maximum height of 
a significant peak and thus, the threshold $\lambda$ that permits to distinguish real peaks from insignificant ones. The same setting is required in the case of end points.

The proposed definition overcomes the dependency of the lowering decision to the dynamic change that occurs on iteratively thinned image. We propose in Subsection 2.2 a hypothesis test framework for the lowering decision in order to set the parameter $\lambda$ to an appropriate threshold.

\subsection{Hypothesis test framework for the lowering decisions}

We propose in this subsection a statistical presentation of the lowering decision. Based on a test significance level $\alpha$, the thinning setting turns out to become locally adjusted and thus, less userdependent than initial globally and manually chosen $\lambda$. For this purpose, we identify 2 different contexts in the lowering process. The first one involves peak and end pixels for which the low neighborhood forms a unique connected component $C$. The second one considers crest pixels for which dark neighborhood is composed of $\mathcal{K}$ components $(2 \leq \mathcal{K} \leq 4)$. We build a statistical test based on a unique hypothesis for peak and end lowering decision, while we construct multiple hypotheses test for the second case in order to take into account the varying size and number of components.

\subsubsection{Statistical test for end and peak lowering}

According to the criterion of equation 1 in Definition 6 deciding that $x$ is a lowerable peak (resp. end) is tantamount to say that $x$ has the same statistical behaviour as the connected component $C$ of the low neighborhood $\mathcal{N}_{8}^{<}(x)$. Thereby, $I_{0}(x)$ is seen as a further observation of the sample composed of initial gray levels of the connected component $C$, where $n=|\mathcal{C} \cup\{x\}|$ is the size of the sample $X_{C}=\left(X_{1}, \ldots, X_{n}\right)$ and $I_{0}(x)$ its maximum value.

This criterion can be used as a decision rule of a statistical test where the null hypothesis $\left(H_{0}\right)$ refers to the fact that the central pixel $x$ is a lowerable peak (resp. end) and its alternative hypothesis $\left(H_{1}\right)$ meaning that $x$ is a significant peak (resp. end) and has to be maintained. As the most common behaviour corresponds classically to the null hypothesis, this test will enable us to decide extensively the lowering of insignificant peaks and ends and thus, meeting the requirements of a statistical test design.

Let us denote $P_{H_{0}}$ the statistical behaviour of each variable in the context of $H_{0}$ (x has the same behaviour as $C$ ). Therefore, for a test of level $\alpha$, we have:

$$
\begin{aligned}
P_{H_{0}}\left(\text { decide } H_{1}\right) & =P_{H_{0}}\left(I_{0}(x)-X_{(1)}>\lambda \mid X_{(n)}=I_{0}(x)\right) \leq \alpha, \\
\text { with } X_{(1)} & =\min _{i=1 \ldots n} X_{i} \text { and } X_{(n)}=\max _{i=1 \ldots n} X_{i}
\end{aligned}
$$

Moreover, we assume that image gray levels have a Gaussian distribution since it is the most common and realistic distribution for a large set of digital images. This assumption also applies to the sample $X_{\mathcal{C}}$ under the null hypothesis $\left(H_{0}\right)$ and implies that $X_{i} \sim \mathcal{N}(\mu, \sigma), \forall i=1 \ldots n$. 
In such a context, we propose now to show that the threshold value $\lambda$ allowing to distinguish real peaks and ends from noisy ones can be evaluated directly from the fixed significance level $\alpha$ and the size $n$ of the sample $\mathcal{C} \cup\{x\}(n=9$ for peak configuration and $n=8$ for end configuration as illustrated in Figure 1(b) and (c)).

Noting $X_{i}=\mu+\sigma \cdot Y_{i}$ with $Y_{i} \sim \mathcal{N}(0,1), Y_{(1)}=\min _{i \in C} Y_{i}$ and $Y_{(n)}=\max _{i \in C} Y_{i}$, we can state that:

$$
\begin{aligned}
P_{H_{0}}\left(\text { decide } H_{1}\right) & =P_{H_{0}}\left(X_{(1)}<I_{0}(x)-\lambda \mid X_{(n)}=I_{0}(x)\right)=\alpha \\
& =P_{H_{0}}\left(Y_{(1)}<1 / \sigma \cdot\left[I_{0}(x)-\mu-\lambda\right] \mid Y_{(n)}=1 / \sigma \cdot\left[I_{0}(x)-\mu\right]\right)=\alpha
\end{aligned}
$$

Consequently, the test statistic used according to Definition [6 of lowerable peaks or ends is the minimum statistic of the corresponding sample $X_{C}$ under the knowledge of its maximum gray level $\left(I_{0}(x)\right)$. By using the well-known cumulative distribution of such i.i.d sample [29], Equation 2 becomes:

$$
\begin{aligned}
P_{H_{0}}\left(Y_{(1)}<1 / \sigma \cdot\left[I_{0}(x)-\mu-\lambda\right] \mid Y_{(n)}=1 / \sigma \cdot\left[I_{0}(x)-\mu\right]\right) & =\alpha \Leftrightarrow \\
1-\left(1-F\left(1 / \sigma \cdot\left[I_{0}(x)-\mu-\lambda\right]\right) / F\left(1 / \sigma \cdot\left[I_{0}(x)-\mu\right]\right)\right)^{n-1} & =\alpha \Leftrightarrow \\
(1-\sqrt[n-1]{1-\alpha}) \cdot F\left(1 / \sigma \cdot\left[I_{0}(x)-\mu\right]\right) & =F\left(1 / \sigma \cdot\left[I_{0}(x)-\mu-\lambda\right]\right)
\end{aligned}
$$

where $F$ is the cumulative distribution function of $\mathcal{N}(0,1)$. Let us denote $\tau_{n}(\alpha)=\frac{1}{\sigma} \cdot\left[I_{0}(x)-\mu-\lambda\right]$. Therefore, $\tau_{n}(\alpha)$ is the quantile of $\mathcal{N}(0,1)$ and can be expressed as follows.

$$
\tau_{n}(\alpha)=F_{\mathcal{N}(0,1)}^{-1}\left((1-\sqrt[n-1]{1-\alpha}) \cdot F_{\mathcal{N}(0,1)}\left(1 / \sigma\left[I_{0}(x)-\mu\right]\right)\right)
$$

which leads to the determination of $\lambda$ such as:

$$
\lambda=I_{0}(x)-\mu-\sigma \cdot \tau_{n}(\alpha) .
$$

Finally, according to the expression of $\lambda$ in Equation 3, the criterion of Equation 1 that allows to lower insignificant peaks and ends knowing $X_{(n)=I_{0}(x)}$ becomes:

$$
X_{(1)} \geq \mu+\sigma \cdot \tau_{n}(\alpha)
$$

In order to calculate $\lambda$ at each pixel $x$, we need to estimate $\mu$ and $\sigma$. Firstly, the standard deviation $\sigma$ of the sample could be assimilated to the image noise standard deviation. Under the assumption of a stationary Gaussian noise, we can consider that noise standard deviation $\sigma$ is constant over the entire image. Secondly, $\mu$ is the mean of the sample, and varies depending on $X_{\mathcal{C}}$. The parameter $\mu$ is estimated empirically on $X_{C}$ by $\bar{X}_{C}=1 / n \cdot \sum_{i=1}^{n} X_{i}$.

The hypotheses test framework enabled us to calculate the threshold $\lambda$ of the parametric thinning at each pixel $x$ as a function of noise mean and standard deviation for each $X_{C}$, the size of $C$ and the initial gray level $I_{0}(x)$ of the pixel $x$. The significance level $\alpha$ is used as a standard control for the 
lowering permissions and thus, is the decision error of the lowering process. If the lowering decision for peak or end can be done using a unitary test, the lowering decision for insignificant crest pixels depends on multiple connected components and leads us to consider the fusion of multiple test hypothesis as proposed in subsection 2.2 .2

\subsubsection{Fusion of multiple hypotheses test for crest lowering}

According to Definition 3, the criterion that allows to lower a crest pixel $x$ states that among his $\mathcal{K}$ darker neighboring components, named $C_{k}$ for $k=1 \ldots \mathcal{K}$, at least $(\mathcal{K}-1)$ verify:

$$
I(x)-\min \left\{I\left(C_{k}\right)\right\} \leq \lambda
$$

In the case of lowerable crests, $\mathcal{K}$ unitary statistical tests can be designed similarly to the one for peak and end. For each unitary test, the null hypothesis $H_{0 k}$ is tantamount to state that $I(x)$ is similar to the current gray levels of pixels in $\left(C_{k}\right)_{k=1 \ldots \mathcal{K}}$. Moreover, as for peak and end hypothesis test, each unitary test is based on a threshold $\lambda_{k}$ that depends on the cardinality $n_{k}$ of $C_{k} \cup\{x\}$ and on the chosen significance level of the test $\alpha_{k}$. Hence, we have:

$$
P_{H_{0 k}}\left(\text { decide } H_{1 k}\right)=P_{H_{0 k}}\left(I(x)-X_{(1)}^{k}>\lambda_{k} \mid X_{\left(n_{k}\right)}^{k}=I(x)\right)=\alpha_{k}
$$

where $X_{(1)}^{k}=\min _{i \in C_{k}} X_{i}$ when knowing $X_{\left(n_{k}\right)}^{k}=I(x)$ are independent variables, since $C_{k} \cap C_{k^{\prime}}=$ $\emptyset, \forall k, k^{\prime}=1, \ldots, \mathcal{K}$.

In order to construct a global test for $\lambda$-crest lowering, we focus on the fusion of such unitary tests. The global null hypothesis $\left(H_{0}\right)$ refers to the fact that no more than one component $C_{k}, k=1 \ldots \mathcal{K}$ is at a distance to $I(x)$ greater than its corresponding threshold $\lambda_{k}$. Therefore:

$$
H_{0}=\bigcup_{i=1}^{K} \bigcap_{k \in K, k \neq i}^{K} H_{0 k}
$$

This decomposition allows us to assert that a fusion of multiple hypotheses should substitute the global null hypothesis $H_{0}$, and in that case, setting $\alpha$ as the significance level of the multiple tests is not the appropriate way to calculate $\tau_{n_{k}}$ for each $H_{0 k}$. Hence, the formula of this decomposition enables us to establish which fusion test to use and thus, which significance level $\alpha_{k}$ to choose for $H_{0 k}$.

The alternative hypothesis $\left(H_{1}\right)$, for its part, implies that 2 components or more are above their respective threshold. Therefore, the level $\alpha$ can be defined such as:

$$
P_{H_{0}}\left(\text { decide } H_{1}\right)=1-P_{H_{0}}\left(S_{\mathcal{K}} \leq 1\right)=P_{H_{0}}\left(S_{\mathcal{K}} \geq 2\right)=\alpha
$$

with

$$
S_{\mathcal{K}}=\sum_{k=1}^{\mathcal{K}} \delta_{k} \text { and } \delta_{k}=\mathbb{1}_{\left\{I(x)-X_{(1)}^{k}>\lambda_{k} \mid X_{\left(n_{k}\right)}^{k}=I(x)\right\}} .
$$


The test statistic $S_{\mathcal{K}}$ is the sum of binary independent random variables $\delta_{k}$ based on independent variables $X_{(1)}^{k}$ knowing that $X_{\left(n_{k}\right)}^{k}=I(x)$. We assume that the global null hypothesis $H_{0}(x$ is a lowerable crest pixel) and the unitary null hypothesis $H_{0 k}$ have the same effect on the law of each statistic $\delta_{k}$, which could be formulated $\forall k=1 \ldots \mathcal{K}$ as follow:

$P_{H_{0 k}}\left(I(x)-X_{(1)}^{k}>\lambda_{k} \mid X_{(n)}^{k}=I(x)\right)=P_{H_{0}}\left(I(x)-X_{(1)}^{k}>\lambda_{k} \mid X_{(n)}^{k}=I(x)\right)=P_{H_{0}}\left(\delta_{k}=1\right)=\alpha_{0}$.

Therefore, $S_{\mathcal{K}}$ follows a binomial law.

$$
S_{\mathcal{K}} \sim \mathcal{B}\left(\mathcal{K}, \alpha_{0}\right) \quad \text { and }, \quad P_{H_{0}}\left(\text { decide } H_{1}\right)=1-\left(1-\alpha_{0}\right)^{\mathcal{K}}-\mathcal{K} \cdot \alpha_{0} \cdot\left(1-\alpha_{0}\right)^{\mathcal{K}-1}=\alpha .
$$

According to Equation [5] for each global test level $\alpha$, we can calculate the unitary test level $\alpha_{0}$ depending on the $\mathcal{K}$ number of components. Figure 11 represents $\alpha$ as a function of $\alpha_{0}$ for the different possible values of $\mathcal{K}$.

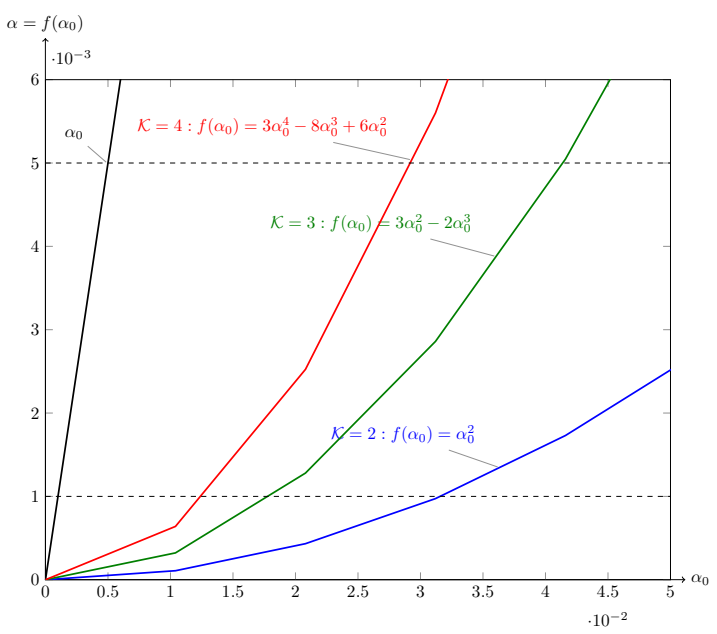

Figure 11: Plot of $\alpha=f\left(\alpha_{0}\right)$. In blue $\mathcal{K}=1$. In green, $\mathcal{K}=2$. In red, $\mathcal{K}=3$.

We note in this plot that we penalize the configurations where $\mathcal{K}$ increases, which means that we are more selective when maintaining junction pixels $(\mathcal{K}=3,4)$. In fact, when $\alpha=0.001$, the adequate unitary test level to choose is $\alpha_{0}=0.032$ for $\mathcal{K}=2$ while it corresponds to $\alpha=0.012$ for $\mathcal{K}=4$. The smaller the unitary test level $\alpha_{0}$ is, the bigger $\tau_{n}$ is and the more lowering decision is taken.

Consequently, for a fixed global test level $\alpha$, we can precalculate the unitary levels $\alpha_{0}=f(\alpha, \mathcal{K})$ as presented in Table 1. It summarizes for some classic significance level values the corresponding $\alpha_{0}$. 
Table 1: Computation of $\alpha_{0}$ as a function of $\alpha$ and $\mathcal{K}$ connected components.

\begin{tabular}{|r||c|c|c|c|c|c|c|}
\hline $\mathcal{K}$ & $5.10^{-2}$ & $10^{-2}$ & $5.10^{-3}$ & $10^{-3}$ & $10^{-4}$ & $10^{-5}$ & $10^{-6}$ \\
\hline \hline 2 & 0.2236 & 0.1000 & 0.0700 & 0.0316 & 0.0100 & 0.0032 & 0.0010 \\
3 & 0.1354 & 0.0589 & 0.0414 & 0.0184 & 0.0058 & 0.0019 & 0.0007 \\
4 & 0.0976 & 0.0419 & 0.0294 & 0.0130 & 0.0040 & 0.0010 & 0.0004 \\
\hline
\end{tabular}

As previously defined, threshold $\lambda_{k}$ corresponds finally to:

$$
\lambda_{k}=I(x)-\mu-\sigma \cdot \tau_{n_{k}}\left(\alpha_{0}\right)
$$

where $n_{k}=\left|C_{k}\right|$ and $\tau_{n_{k}}$ is the quantile that can also be precalculated as:

$$
\tau_{n_{k}}\left(\alpha_{0}\right)=F_{\mathcal{N}(0,1)}^{-1}\left(\left(1-\sqrt[n_{k}-1]{1-\alpha_{0}}\right) \cdot F(1 / \sigma \cdot[I(x)-\mu])\right) \text {. }
$$

Finally, the thinning no longer depends on a global parameter $\lambda$ but on the test significance level $\alpha$ and the noise standard deviation $\sigma$. The test level expresses a choice made by the user to maintain significantly contrasted configurations. The proposed statistically adjusted thinning is called in the sequel Self Contrast Controlled Thinning (SCCT).

\subsubsection{SCCT in the general noise context of tabulated distribution}

We presented in the previous subsection the calculus of lambda based on the assumption of a Gaussian noise distribution. Our aim here is to generalize this calculus for any tabulated noise law that is consequently parameterized.

Let us denote by $g$ the pivotal quantity of the distribution. The function $g$ permits to standardize the studied sample $(\{x\} \cup C)$. The function $g$ is bijective on the set of values taken by the variables $\left(X_{i}\right)_{i=1 \ldots n}$ and we could write $Y_{i}=g\left(X_{i}\right)$.

If $g$ is an increasing function, we could write the Equation 2 as follows:

$$
\begin{aligned}
P_{H_{0}}\left(\text { decide }_{1}\right) & =P_{H_{0}}\left[X_{(1)}<I_{0}(x)-\lambda \mid X_{(n)}=I_{0}(x)\right] \\
& \left.=P_{H_{0}}\left[g\left(X_{(1)}\right)<g\left(I_{0}(x)\right)-\lambda\right) \mid g\left(X_{(n)}\right)=g\left(I_{0}(x)\right)\right] \\
& \left.=P_{H_{0}}\left[Y_{(1)}<g\left(I_{0}(x)\right)-\lambda\right) \mid Y_{(n)}=g\left(I_{0}(x)\right)\right]
\end{aligned}
$$

Therefore, we still exploit the law of $\min \mid \max$ statistic in the decision error as written hereafter:

$$
P_{H_{0}}\left(\text { decide }_{1}\right)=\left[1-\frac{\left.F\left(g\left(I_{0}(x)\right)-\lambda\right)\right)}{F\left(g\left(I_{0}(x)\right)\right)}\right]=\alpha,
$$

where $F$ is the cumulative distribution of the standardized sample.

Consequently, the statistical adjustment of the SCCT method could be extended to other 
types of noise distribution for which the cumulative distribution can be tabulated and the pivotal quantity is known.

In this work, we take the example of a Gaussian noise distribution with $Y_{i}=g\left(X_{i}\right)=\frac{X_{i}-\mu}{\sigma}$. Another example could be a more complex model of Poisson-Gaussian noise distribution encountered in astronomy [30], medicine [31] and biology [32].

As explained by Makitalo et al. [33], a generalized Anscombe transformation [34] can be used to stabilize the variance of this distribution. This transformation corresponds to the pivotal quantity of the noise distribution.

The variance stabilization approach consists in two main steps. Preliminarily, the authors define the resulting pixel intensity $X$ corrupted by Poisson-Gaussian noise as follows:

$$
\stackrel{*}{X}=\eta P+\stackrel{*}{N}
$$

where $P \sim \mathcal{P}(y)$ and $\stackrel{*}{N} \sim \mathcal{N}\left(\mu, \stackrel{*}{\sigma}^{2}\right)$.

The first step is a standardization of the variable $X$ by the following equation:

$$
X=\frac{\stackrel{*}{X}-\mu}{\eta}, \sigma=\frac{*}{\eta}
$$

At this stage, the standardized variable corresponds to a specific Poisson-Gaussian variable as presented in Equation 15;

$$
X=P+N,
$$

for which $P \sim \mathcal{P}(y)$ and $N \sim \mathcal{N}\left(0, \sigma^{2}\right)$.

The second step consists in applying a generalized Anscombe transformation [34] to $\stackrel{*}{X}$ in order to stabilize the variance of the variable $\left(\operatorname{var}\left(X^{\prime}\right) \approx 1\right)$. Hence, by injecting the standardization of Equation 14 to the Ascombe transformation of $X$, we have:

$$
X^{\prime}=f_{\sigma}(X)=\left\{\begin{array}{ll}
2 \sqrt{X+3 / 8+\sigma^{2}}, & X>-3 / 8-\sigma^{2} \\
0, & X \leq-3 / 8-\sigma^{2}
\end{array} .\right.
$$

Finally, the pivotal quantity of a Poisson-Gauss distribution corresponds to Equation 16. In conclusion, if the standardization and tabulation of a noise distribution are possible, one may exploit the proposed statistical adjustment of the parametric thinning by estimating the specific noise parameters.

\subsection{Implementing hierarchical queues for the self contrast controlled thinning}

A hierarchical Queue is a set of ordered FIFO (First In First Out) queues and was implemented for watershed in [35]. Each queue has a priority level that can be linked to the gray levels of an image. In 
HQs, popping is possible only from the highest priority queue while pushing is possible on each queue.

The first push in a HQs is crucial since it defines the higher priority level of the first pop. If other pushes of higher priority (lower gray value) than the first one are needed during the execution, an "emergency queue" is created. In the emergency queue, the priority of gray levels is not respected during the treatment since it contains all pixels of low gray levels compared to the first push. Its use should be limited to respect the ascendant treatment required for the parametric thinning and the SCCT.

The library Pink has implemented the HQs to quicken the parametric thinning $\lambda$-Skeleton in the same way as watershed, starting from regional minima and propagating the lowering process through their neighbors. The ascendant treatment of $\lambda$-lowerable pixels is not totally ensured as required in the appendix of [23] since all neighbors of lowerable pixels are added with no test on their "deletability".

To ensure an ordered treatment of the lowerable pixels, we add a first review of the image that initializes the HQs with current $\lambda$-lowerable pixels. When popping a pixel (if a lowering is carried out), the HQs is updated by adding the newly $\lambda$-lowerable neighbors. Hence, this ensures the systematic examination of the darkest lowerable pixels and decreases the risk of disconnections. The operating mode we adopted has reduced the number of lowering instructions compared to Pink $\lambda$-Skeleton as described in [27].

\section{Experimental results}

Several evaluation protocols have been proposed for binary skeletonization [36]-[41], but to the best of our knowledge, very few ones are dedicated to the skeletonization of gray images [42, 43]. These protocols, in both cases, consider the binary skeleton graph (not the gray skeleton) and intend to validate its fundamental properties, such as homotopy [41, 39], geometry preservation [37, 6, 39, 42, 43, 41], robustness to noise [38, 40, 6] and to rotation [40].

In this section, we compare the behaviour of the proposed SCCT to Steger's detector of curvilinear structures [1] and to the $\lambda$-Skeleton of Pink library [23]. This evaluation is performed quantitatively on synthetic images followed by illustrations on real ones. To this purpose, we focus on the skeleton quality according to its fundamental properties.

Therefore, Subsection 3.1 is devoted to the parameters setting of the skeletonization methods concerned by this evaluation. Subsection 3.2 is dedicated to the description of the objective evaluation. Finally, in Subsection 3.3, we illustrate the interest of implementing the SCCT on real images from biometrics, letter recognition and biomedical imaging applications. We present the acquired images for each application, explain their respective requirements, adjust their parameters and finally discuss the obtained results. 


\subsection{Parameters setting of the compared skeletonization methods}

Several algorithms are proposed in the literature to implement the skeletonization. We identify two main classes: pixel-based methods such as thinning and sub-pixel-based methods such as the differential ones 1] 44] or the distance map transforms [45]. Therefore, we propose to compare our pixel-based method SCCT to methods with proven efficiency from each class. The first one is the Differential Line Detector (DLD) [1] and the second one is the $\lambda$-Skeleton of [23].

We present in this subsection the parameters setting of both DLD and $\lambda$-Skeleton methods.

\subsubsection{Differential Line Detector method DLD}

The DLD algorithm [1] models the image as a surface and finds ridge points in sub-pixel accuracy for low image resolution, based on directional derivatives. In fact, the direction maximizing the concave curvature of the gradient image is used to localize crest points. In order to calculate the first and second derivatives of the image, convolution with Gaussian kernels of scale $\sigma$ are used with respect to objects lines width.

A linking step of crest points is then performed, guided by the tangent direction at each point. The linking algorithm starts at points having a second directional derivative (the tangent direction) larger than a parameter high, and follows lines until the second directional derivative is smaller than another parameter low or until a junction point is found.

Therefore, 3 parameters have to be set for the $D L D$ method: the scale $\sigma$ and the low and high hysteresis thresholds. We use in this work the parameters setting suggested by the author in [1] exposed in table 2. We note that the $D L D$ method does not put constraints on the object connectedness and assumes that all the object lines have similar widths.

\subsection{2. $\lambda$-Skeleton of Pink library}

As presented in Section 1. $\lambda$-Skeleton uses a global parameter $\lambda$ that constraints the lowering of insignificant branches, extremities and peaks. We propose to set this parameter using the mean value of the lowering threshold $\tau_{n}(\alpha)$ (as tabulated in Section 2) and the noise standard deviation according to the formula: $\lambda=\overline{\tau_{n}(\alpha)} \cdot \sigma_{\text {noise }}$. These values are well adapted to the method, since a greater $\lambda$ value eliminates more end points but disconnects the object, while smaller $\lambda$ values create further connections, and thus, unsuitable characterization of the objects.

Table 2 summarizes the parameters setting for each skeletonization method and indicates how dependent they are on user intervention.

\subsection{Quantitative evaluation on synthetic images}

In 1987, Haralick initiates an outline of a quantitative evaluation protocol for binary thinning methods [36]. The author evaluates the behaviour of thinning on noisy data and proposes to conduct 
Table 2: Parameters setting for the considered methods in the evaluation protocol.

\begin{tabular}{clll}
\hline Method & $\begin{array}{l}\text { Parameters set } \\
\text { by the user }\end{array}$ & Description & Setting \\
& $\sigma$ & standard deviation of the gaussian kernel & $\sigma=\sqrt{3} \cdot w$ \\
DLD & low & First hysteresis threshold & {$[0.2 h i g h, 0.5 h i g h]$} \\
& $h i g h$ & Second hysteresis threshold & $h i g h=h \cdot\left|g_{w}^{\prime}-g_{-w}^{\prime}\right|$ with \\
& $\lambda$ & sets the significant height of skeleton lines ac- & $\lambda=\overline{\tau_{n}(\gamma)} \cdot \sigma_{n o i s e}$ \\
\hline Pink & $\lambda$ & cording to noise and contrast & \\
\hline \multirow{2}{*}{ SCCT } & $\alpha$ & test significance level, sets the threshold dis- & $\alpha \in\left\{0.05,0.01, . .10^{-6}\right\}$ \\
& & tance between skeleton lines and background & \\
& & Noise standard deviation & $\widehat{\sigma}_{n o i s e}$ \\
\hline
\end{tabular}

an evaluation protocol in 3 main steps: specifying the input data model in an ideal case, choosing the distorsion that reflects the evaluated property and finally proposing the corresponding quality measure. In this work, we follow a slightly different outline to design an evaluation protocol for gray skeletonization methods. In fact, we assume that gray images present more challenging situations than binary ones (the most commonly used distorsion for binary images is the contour distorsion). We propose here the modeling of synthetic images according to some challenging situations, before proceeding with the classical distorsions such as noise addition. These typical configurations maybe related to contrast and line widths variation, high curvature, intersections, free extremities etc..

Therefore, in Subsubsection 3.2.1 we address the issue of constructing synthetic images in order to evaluate the results of skeletonization methods in the challenging situations. Once generated, these images are corrupted by an additive Gaussian noise. In order to accurately evaluate the resulting skeletons, we present in a second subsection the measures chosen from the literature to evaluate the robustness to noise, the homotopy and the ability to preserve geometry of initial objects. The third subsection is devoted to an objective evaluation when the reference skeletons are known and with a final discussion on the performances.

\subsubsection{Generation of synthetic images}

For an accurate modeling of the synthetic images, we focus on the challenges of gray skeletonization. Interconnected lines, different line widths, high curvatures and presence of noise are the main challenging situations according to which we model the synthetic images in order to validate the fundamental properties of skeletonization. To this end, we generate synthetic images from dilated reference skeletons using disk structuring elements of various sizes in order to obtain gray objects that meet the typical circumstances cited above.

First, the rings gray image of Figure 12 (d) is derived from the skeletal rings of Figure 12 (a). These 
objects have no extremity and intend to validate the detection of curved and closed lines. We dilate the binary skeleton using specific disk radiuses (binary disks) for each ring (from the smallest to the greatest ring of radius 9,4 pixels). Then we dilate the resulting image using gray disk structuring elements of respective radiuses 5, 4 and finally superpose the skeleton (turned to gray) to obtain the final gray rings. Superposing the gray skeleton to the gray dilated rings allows to position central crest lines and generate an image profile that resembles to a smooth topographic relief.

Second, the network skeleton image of Figure 12,(b) contains interconnected lines with other free end branches. We use this image to test the ability of the skeletonization methods to preserve the connectedness of objects and their extremities. The gray network image is obtained through consecutive gray dilations (consecutive gray disks of radiuses 5,2 ) with a final superposition of the skeleton turned to gray (Figure 12 (e)).

Third, the simple intersection image presents crossing lines of different widths. This image intends to verify the ability of the skeletonization methods to detect crossing lines with various widths. The skeleton image is composed of two separate horizontal and vertical line images. The dilations of both of them is made individually using different gray disks. For the vertical line, we use $r=3,2$ while for the horizontal line, we choose the radiuses $r=9,5$.

For the three images, we used a uniform background gray level equal to 25 in order to avoid truncation of low noise values. We indicate in the Figure 12 the difference $D$ of average gray levels between the foreground and the background.

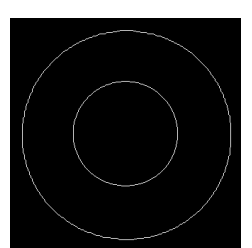

a: Rings skeleton $326 \times 326$

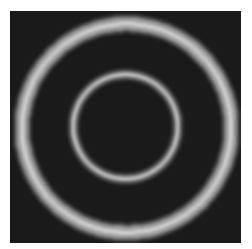

d: $D=81$

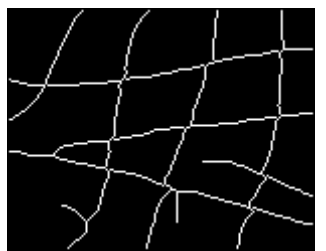

b: Network skeleton

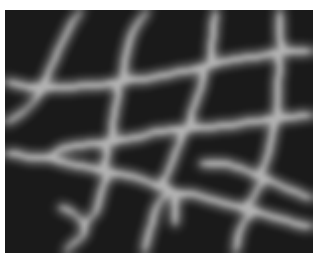

e: $D=46$

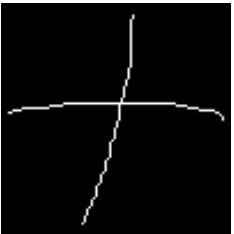

c: Simple Intersection skeleton

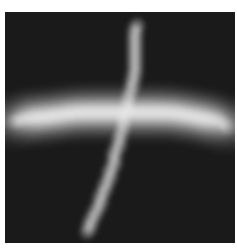

f: $D=65$

Figure 12: Synthetic images construction: From binary skeleton to gray level images.

\subsubsection{Evaluation criteria and measures}

We based our evaluation protocol on criteria linked to properties of binary skeletons. In fact, even if image treatment is different between gray and binary skeletonizations, the objectives of this 
operation are not. Therefore, we assess that the classical binary skeleton properties i.e homotopy, geometry preservation and robustness to noise are the properties to validate in gray case. We use the buffer-method for geometry preservation property and count endpoints and connected components for homotopy evaluation. We successively present below the chosen measures for the evaluation criteria.

Buffer method to evaluate the geometry preservation. The buffer method consists in validating the accuracy in location of real skeleton pixels regarding the ideal skeleton, with a certain tolerance. This measure is similar to the Area method used in [5]- 6]- [37]- 41] that calculates the overlap percentage between the dilated real skeleton and the dilated ideal one. First, a dilation is operated on the reference or the real skeleton to calculate respectively the matched extraction or matched reference. The structuring element is a disk of a radius that corresponds to half-width of the object to adjust the tolerated displacement of the skeleton. Once dilated, three indicators are calculated. The True Positive $T P$ is the number of successfully matched extraction. The False positive $F P$ is the number of wrongly extracted skeleton pixels, while False Negative $F N$ defines the missing reference in the detection. These indicators are illustrated by Figure 13 for a simple straight line. Finally, the completeness and correctness measures are calculated to assess the geometrical accuracy of the real skeleton compared to the reference one.

The completeness is the percentage of the matched extraction regarding the total reference

$$
C p=T P /(T P+F N)
$$

The correctness is the percentage of the matched extraction regarding the total extraction

$$
C r=T P /(T P+F P)
$$

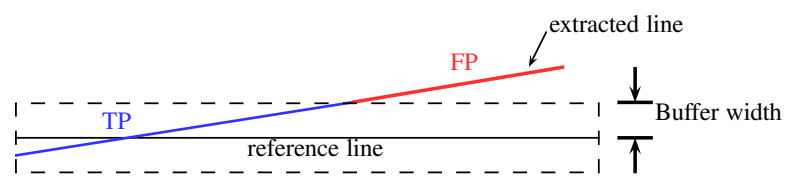

matched extraction

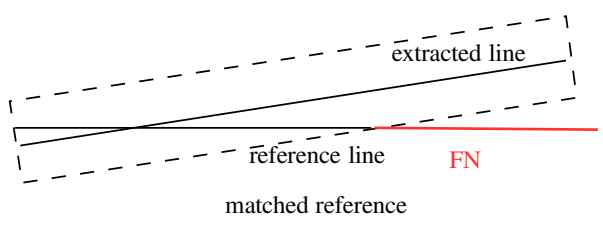

Figure 13: Matched reference and extraction for the buffer method on a simple straight line skeleton 
is the preservation of the topological characteristics as explained in Subsection 1.1. Therefore, a counting of the foreground and background components, in addition to a counting of skeleton endpoints permit us to check if the skeletons obtained by the 3 methods preserve

For B.C.C, O.C.C and E, negative ratios correspond to the appearance of extra and insignificant information while positive values correspond to missing information in the real skeleton. For the sake of clarity, large ratios deviations (respectively small) are set to 5 (respectively -5 ) values. Finally, a score $S$ is used to evaluate the overall performance of the methods:

$$
S=1-[((1-C p)+(1-C r)+(|O . C . C| / 5)+(|B . C . C| / 5)+|E| / 5)] .1 / 5
$$

Measures of B.C.C, O.C.C and E are normalized in the formula because of possible divergence of the results and the imposed limits. According to this formula, the best scores should be close to one.

\subsubsection{Results of the objective evaluation}

After establishing our evaluation methodology, constructing the synthetic images, choosing the criteria and the corresponding measures, we proceed in evaluating the 3 skeletonization methods by varying noise level and then, computing the quantitative measures for each result. The resulting measures are presented in Figure 14 for the network, rings and intersection synthetic images.

We first discuss the results according to geometry preservation criteria. The measures of $C p, C r$ in Figure 14 are maximal for DLD. In fact, as illustrated in Figures 15] and 16, the DLD skeleton is smooth for the 3 images and its quality is not affected neither by contrast changes, incremented noise standard deviation nor high curvature. Skeleton lines are correctly positioned on center lines and this is induced by the smoothing step performed before the crest points detection process. For Pink library implementation of the $\lambda$-Skeleton, we note lower performance of the method (for its two options) compared to the proposed SCCT. In fact, the first option (removing all ends and peaks) affects the completeness $C p$ of the skeleton. The second option (keeping ends and removing all peaks) affects the correctness $\mathrm{Cr}$ of the skeleton since the method detects insignificant/spurious branches. The effect of both options is also reflected by the homotopy criterion that consists in counting endpoints $(E)$. The impact of activating/desactivating $\lambda$-Skeleton options is noticeable especially for the rings image 
indicators of Figure 14 and is illustrated in Figure 15. The rings image has the highest deviation ${ }_{530}(\bar{D}=127)$ between object and background. This deviation affects the progression of the lowering decision from the background to the spurious branches of object, even for the highest values of $\lambda$.
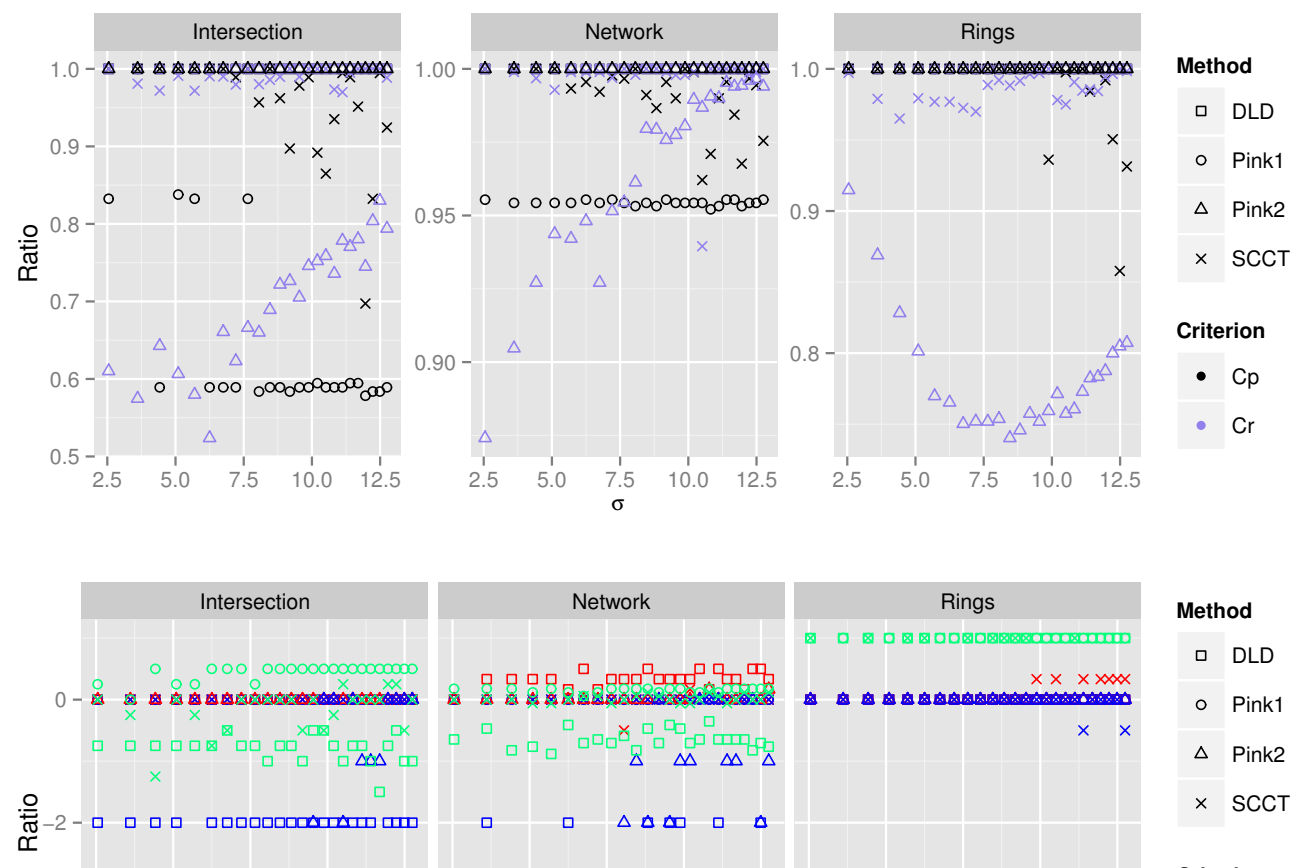
Method
口 DLD
○ Pink1
$\triangle$ Pink2
$\times$ SCCT
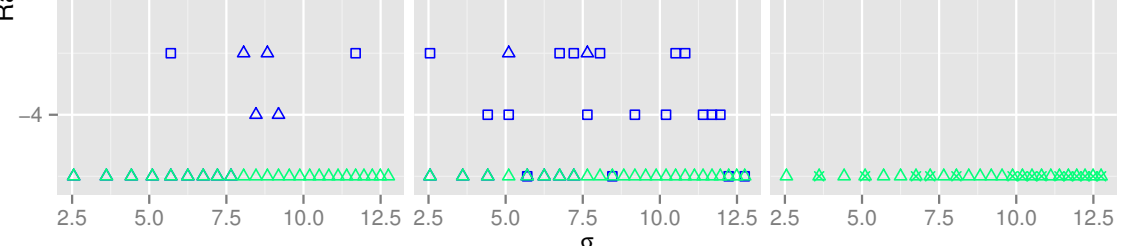

Criterion

- BCC

- $E$

- OCC

Figure 14: Evaluation graphs for the 3 synthetic images using 5 quantitative measures. Noise varying from $\sigma_{\text {noise }}=2$ to 12 by a step of 0.5 . Measures: $C_{p}$ Completeness, $C_{r}$ Correctness, $Q$ Quality calculated for a 5 pixels buffer width, B.C.C Background connected components (4-connectivity), O.C.C: Object Connected Components (8-connectivity) and $E$ End points. Methods evaluated are: (Pink1): No end and no peaks are maintained. (Pink2):Peaks are removed and high ends are maintained. (DLD): Differential Line Detector. (SCCT): Self Noise and Contrast Controlled Thinning.

Second, we focus on the measures linked to homotopy preservation, (O.C.C, B.C.C, E). According to these indicators, SCCT appears to be a compromise between correctly detecting the connected components of the background/foreground, and preserving the significant extremities. On one hand, DLD's indicators for object and background connectedness are the less accurate compared to Pink's results and to the SCCT ones. This is explained by the fact that differential methods do not put constraints for homotopy and assume that all object lines have similar widths. The linking step that aims to connect sub-pixel crest lines fails when object lines widths are different. On the other hand, 
results based on Pink library diverges from the expected results concerning the extremities preservation

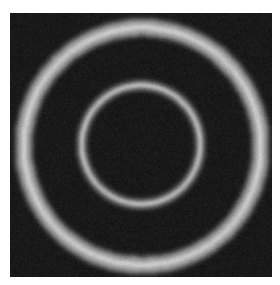

Add noise $\sigma=5.1$

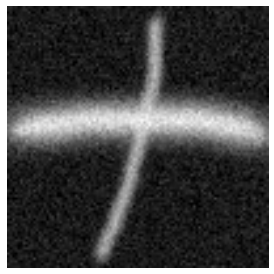

Add noise $\sigma=12.49$

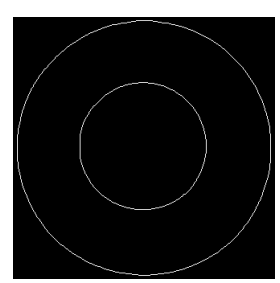

DLD

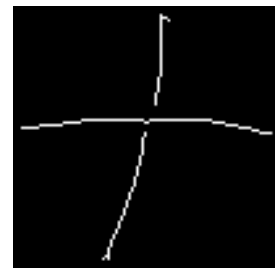

DLD

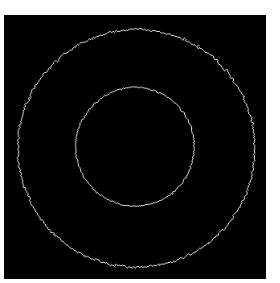

SCCT: $\alpha=10^{-6}$

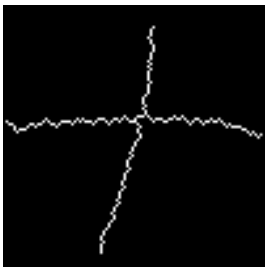

SCCT: $\alpha=10^{-4}$

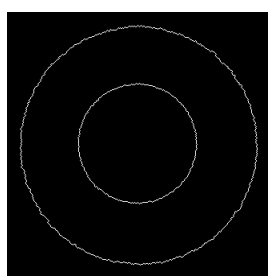

Pink1 $\lambda=61.2$

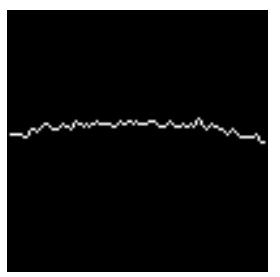

Pink1

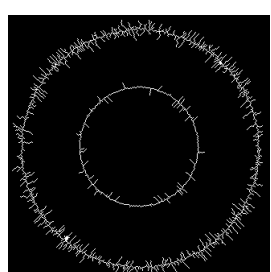

$\operatorname{Pink} 2 \lambda=51$

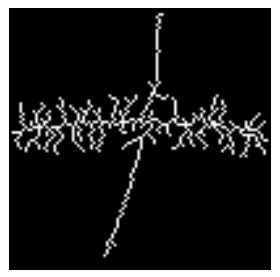

Pink2

Figure 15: Results on the network synthetic image. The mean gray level deviation is $D=46$ and, additive white Gaussian noises is applied with: $\sigma=12.49$. Pink1: parametric thinning removing all ends and peaks. Pink2: parametric thinning removing all peaks and maintaining highly contrasted ends.

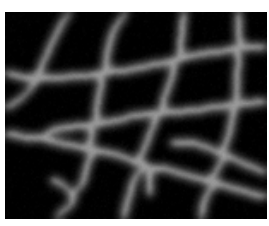

Add noise $\sigma=2.55$

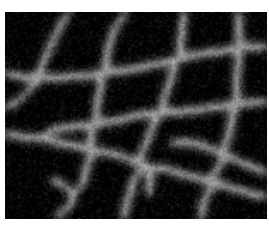

Add noise $\sigma=12.49$

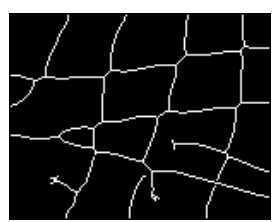

DLD

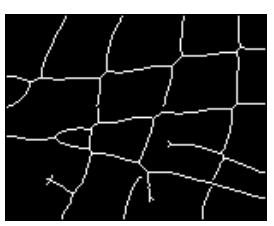

DLD

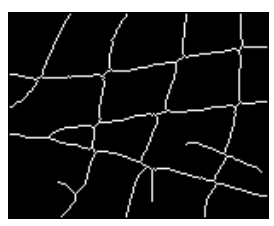

$\operatorname{SCCT}\left(\alpha=5.10^{-2}\right)$

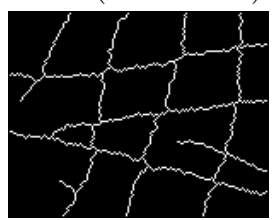

$\operatorname{SCCT}\left(\alpha=5.10^{-2}\right)$

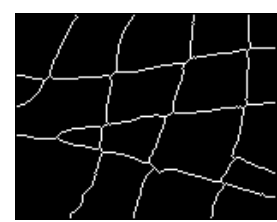

Pink1

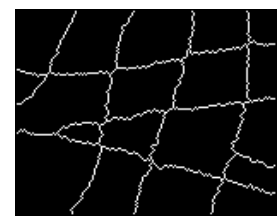

Pink1

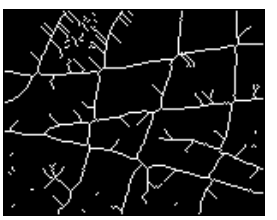

Pink2

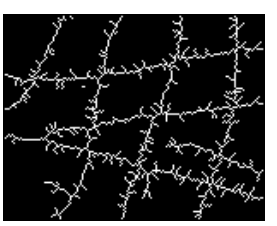

Pink2

Figure 16: Results on the network synthetic image. The mean gray level deviation is $D=46$ and, an additive white Gaussian noises is applied with successively: $\sigma=2.55$ and $\sigma=12.49$.

After discussing separately the accuracy of each method results according to geometry and homotopy preservation, we suggest to evaluate the global performance of the skeletonization methods. We find out that the SCCT method is stable according to both geometry and homotopy indicators. Table 3 details the average values of the evaluation measures as calculated on $N=60$ noisy images.

${ }_{545}$ This table shows that SCCT spatial positioning is correct, that its homotopy indicators are close to zero compared to Pink and DLD and that its results are less impacted by disruptions, high curvatures, 
changing line widths, contrast and free extremities. According to SCCT obtained skeletons, we note

Table 3: Average indicators values for each method, computed with the 3 synthetic images measures.

\begin{tabular}{|c|c|c|c|c|c|c|}
\hline Method & $\overline{C_{p}}\left(C_{p \text { ideal }}=1\right)$ & $\overline{C_{r}}\left(C_{r \text { ideal }}=1\right)$ & $\overline{O . C . C}\left(O . C . C_{\text {ideal }}=0\right)$ & $\overline{B . C . C}\left(B . C \cdot C_{\text {ideal }}=0\right)$ & $\bar{E}\left(E_{\text {ideal }}=0\right)$ & $S$ \\
\hline DLD & 1.00 & 1.00 & -1.81 & 0.11 & -0.15 & 0.92 \\
\hline SCCT & 0.97 & 0.98 & 0.01 & 0.03 & 0.17 & 0.98 \\
\hline Pink1 & 0.86 & 1.00 & 0 & 0 & 0.19 & 0.96 \\
\hline Pink2 & 1.00 & 0.81 & -3.89 & 0.003 & -5.00 & 0.61 \\
\hline
\end{tabular}

that the adequate test significance level $\alpha$ used for the implementation is linked to respective images contrast quantified by the average distance $D$ between background and foreground gray values. In fact, for the rings image, this distant is the biggest $(D=81)$ and its corresponding test level is the smallest $\left(\alpha=10^{-6}\right)$, while for the less contrasted image (network $D=45$ ), the largest significance level is used $\left(\alpha=5.10^{-2}\right)$ to better preserve low contrasted branches. This means that SCCT thinning method controls the preservation of low and high contrasted branches via the test significance level which is linked to each image contrast. The next subsection consists of an illustration of the three methods with a slight change of the last method. We choose to ignore all peaks and extremities for a better performance and a better rendering of the method.

\subsection{Illustrations on applications of interest}

In this section, we present real images stemming from major application domains: biometrics, character recognition and medical imaging and discuss the results for each application field according to their respective objectives.

The first application of interest is biometrics based on fingerprint recognition. Indeed, skeletonization is a crucial step of the matching process since it allows to detection of minutiae composed of fingerprint bifurcations and extremities. Numbers and positions of these minutiae are considered as salient features guiding the matching. Therefore, these minutiae correspond to the skeleton nodes and extremities easy to identify on the thin skeleton. To illustrate the performance of our method, we have selected a fingerprint image from the publicly available FVC2000 database 3 , published in [46]. Figure 17 shows an example of such images acquired with a low-cost sensor.

\footnotetext{
${ }^{3}$ http://bias.csr.unibo.it/fvc2000/databases.asp
} 


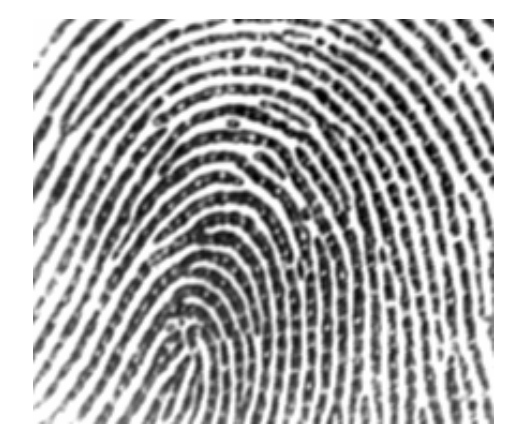

Figure 17: Fingerprint image from the FVC2000 database $(215 \times 189)$.

The second domain of interest is the recognition of characters. In fact, the automatic processing and analysis of large volumes of old documents and manuscripts is an active research domain that uses the skeletonization as a pre-recognition task [47, 48, 49]. We choose to test the skeletonization results on an image from the Persian heritage image binarization dataset 4 that suffers from wide range of degradation. The sample image we use is presented in Figure 18. The processing of this image aims to separate the Persian letters from noisy background containing traces of low contrasted letters and exposed to uneven illumination.

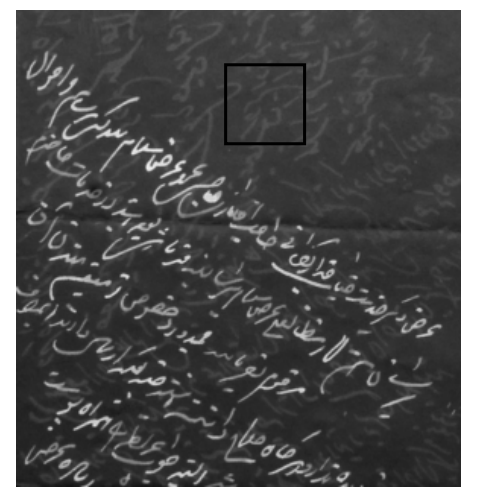

Figure 18: Persian manuscript sample with black box delimiting the region where noise is estimated. $\widehat{\sigma}_{\text {noise }}=7.93$.

The third application presented in this subsection is the biomedical imaging. A growing interest to the morphological tools is noticed in the biomedical imaging field, in particular to the quantification of bone micro-architecture of bone [50]-[52]. This interest is within the scope of Voxelo project funded by th The French National Research Agency5, which objective is the development of new methods

${ }^{4}$ http://www.iapr-tc11.org/mediawiki/index.php/Persian_Heritage_Image_Binarization_Dataset_(PHIBD_2012) 5 http://www.agence-nationale-recherche.fr/en/anr-funded-project/?tx_lwmsuivibilan_pi2\%5BCODE\%5D=ANR-12-TECS-0018 
for early diagnosis of the knee osteoarthritis. To this end, the extraction of the trabecular network is of importance in order to evaluate meaningful morphological parameters such as the number of nodes, segments length and width, etc.. The skeleton of high resolution scanner images (HR-pQCT) should therefore be as accurate as possible. Figure 19 shows a HR-pQCT slice image acquired at the

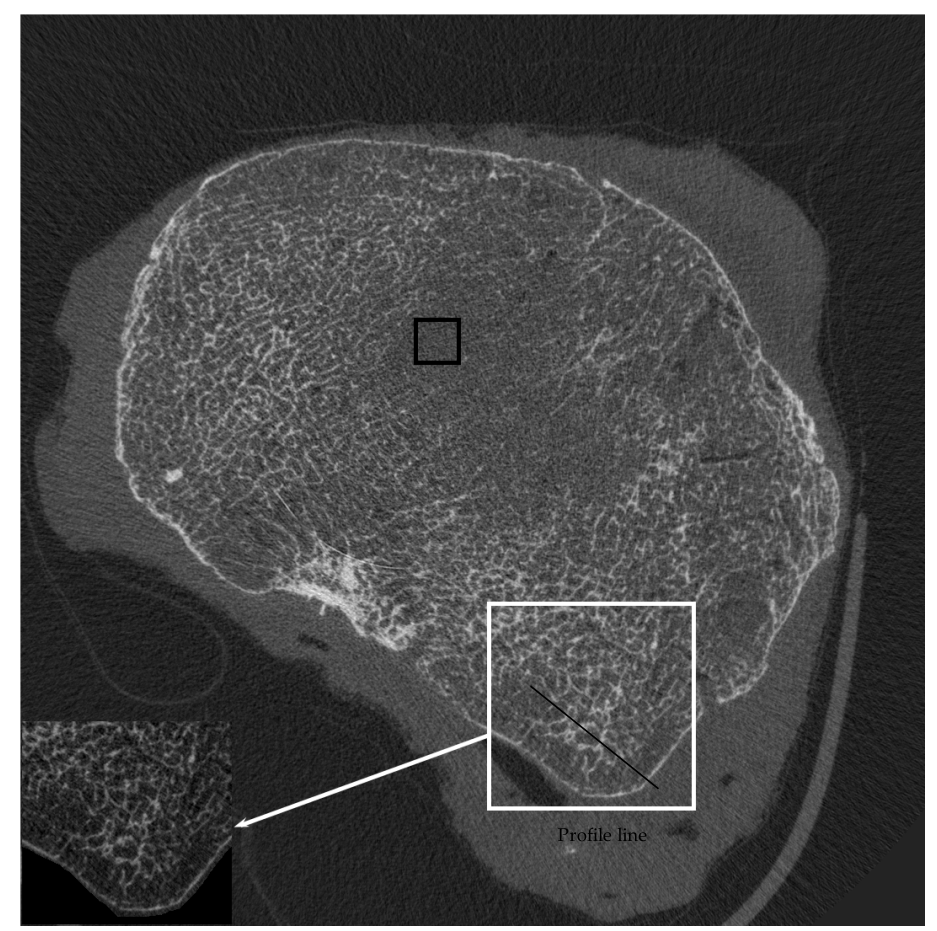

HR-pQCT image $(1400 \times 1400)$ with a selected crop to treat $(249 \times 241)$.

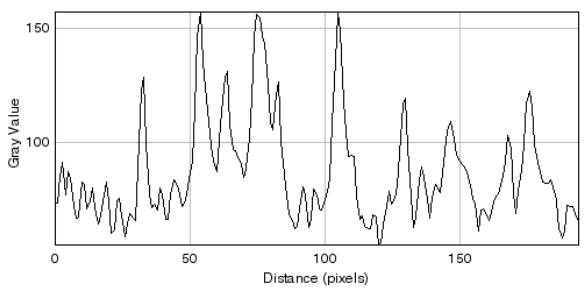

Profile plot on black line from HR-pQCT image.

Figure 19: HR-pQCT image from the Voxelo project. Noise estimated empirically in the black box region.

IMOSAR platform 6 using an Xtrem CTScanco. The challenge of the trabecular structure extraction is the filtering of a textured background and the detection of low-contrasted lines while maintaining

\footnotetext{
${ }^{6}$ IMOSAR is a platform focused on multi-scale imaging derived from X-ray structure of the osteoarticular Federal Research of the Medicine Faculty of Paris Diderot University.
} 
varies from 110 to 150 (on a scale of 255 gray levels) while background gray levels vary from 0 to 120 , which shows the low contrast between the trabecular structures and the background.

For each application image presented in this section, we identify an interesting disruption to overcome. The biometric image of figure 17 presents regular lines highly contrasted and spatially close. The character recognition sample shows Persian letters under varying luminance and superposed to a noisy background containing identical characters. The HR-pQCT trabecular structure has irregular width and low contrast. We discuss the results of the skeletonization methods according to theses particularities and with respect to their objectives consisting namely in maintaining the connectedness of the objects and preserving their extremities and geometry.

Setting the algorithms parameters for the three applications. In order to perform skeletonization on images of Figures 17 [18][19, we need to set respective methods parameters to the adequate values.

First, we need to set the DLD parameters. For the biometric image of Figure 17 the maximum line width is $2 w=4$ pixels and therefore $s=\sqrt{3} . w=1.8$. Similarly we choose $s=1.5$ for the Persian letter of Figure 18 and $s=2.5$ for the HR-pQCT crop image of Figure 19,

Second, the parameters of the SCCT method are: the standard deviation $\sigma$ of the background noise and the significance level of the lowering decision explained in Section 2 , For all these images, the standard deviation $\sigma_{\text {noise }}$ is estimated empirically on the regions we consider as background and the test level is set according to the image contrast. In the case of the fingerprint image of Figure 17, the background noise is located between the minutiae. These minutiae are highly contrasted, thus, the test significance level has to be small $\left(\alpha=10^{-5}\right)$ to target the upper lines only. Besides, the noise on Persian letters is estimated empirically on a region represented by a black box placed on the original Persian manuscript image of Figure 18. Indeed, the characters in the background of Figure 18 are residuals from other pages of the manuscript and are identified as pixels linked to noise. Therefore, for the proposed adjusted thinning, we estimate $\widehat{\sigma}=7.93$ and choose $\alpha=10^{-5}$ for the high-contrasted letters to skeletonize. For the trabecular structure image, the difficulty lies in filtering a textured background and detecting low-contrasted lines. For this application, we estimate the noise on the black box region $(\widehat{\sigma}=4)$ of Figure 19 and choose the highest possible value for the test significance level $\alpha=5 \cdot 10^{-2}$.

The parametric thinning of Pink library uses a manually selected and global parameter $\lambda$ that manages simultaneously noise and contrast. This parameter is estimated using the mean value of the lowering threshold $\tau_{n}(\alpha)$ (see simulations of Section 21) and using the noise standard deviation according to this formula: $\widehat{\lambda}=\overline{\tau_{n}(\alpha)} \cdot \widehat{\sigma}_{\text {noise }}$. Therefore, we choose respectively $\widehat{\lambda}=80,30,16$ for the biometric, character recognition and medical application images. 
Results and Discussion. As noticed in quantitative evaluation, (DLD) results are the smoothest skele-

ton compared to (SCCT) and ( $\lambda$-Skeleton) for Figures 20-21-22 However, (DLD) is non homotopic for the 3 applications. It has difficulties to link lines and to create junctions as shown in Figures 20+21-22, and especially when these lines' widths are irregular as for the bone trabecular structure.

We note that the result of the proposed (SCCT) method is close to DLD, especially in Figures 20 and 21. It appears more disrupted on the bone trabecular structure of Figure 22 since the lines are the less regular and contrasted compared to the other applications. Nevertheless, the adjusted thinning ensures the connectedness of the objects and preserves their extremities.

The results of $\lambda$-Skeleton show the same positioning on the high crest lines as our result with (SCCT). However, in addition to these significant crest lines, ( $\lambda$-Skeleton) keeps other insignificant crests as for fingerprint in Figure 20 and Persian letter in Figure 21. This result suggests that the parameter $\lambda$ is under-estimated. Paradoxically, we note on the same examples, that the foreground insignificant branches persist, which suggests this time around that $\lambda$ is over-estimated. This incoherent result is linked to the global adjustment of the contrast parameter which manages the lowering of $\lambda$-peaks, $\lambda$-ends and $\lambda$-crests simultaneously and without considering the variability of components sizes and numbers. We notice that (SCCT) is the most likely to meet skeleton structural properties that are fundamental for the studied application fields.

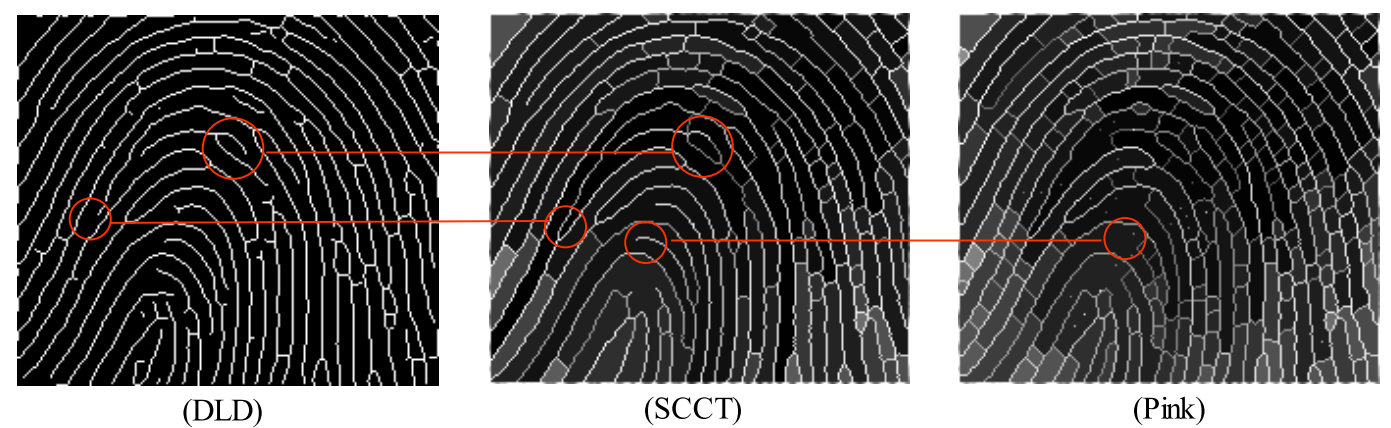

Figure 20: Results of skeletonization on the biometric image. (DLD): $s=1.8$, low $=3$ and high $=6$. (SCCT): $\widehat{\sigma}_{\text {noise }}=16$ and $\gamma=10^{-5}$. (Pink): $\widehat{\lambda}=\overline{\tau_{n}}$. $\widehat{\sigma}_{\text {noise }}=80$. 


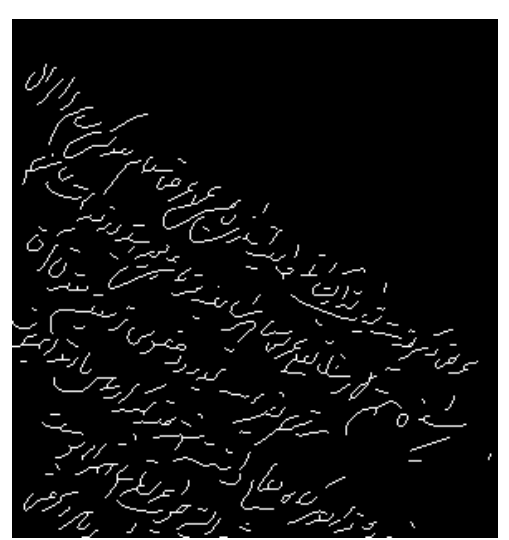

(DLD)

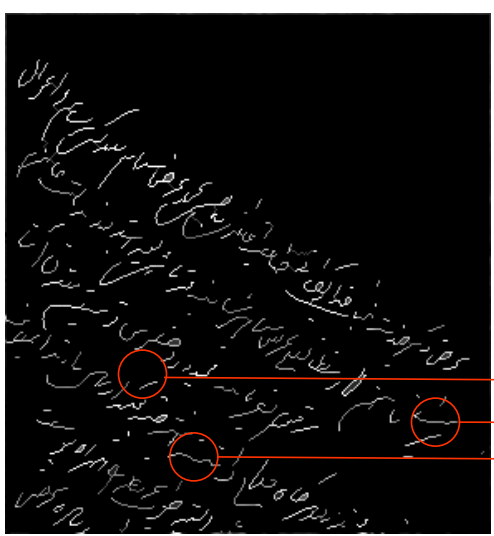

$(\mathrm{SCCT})$

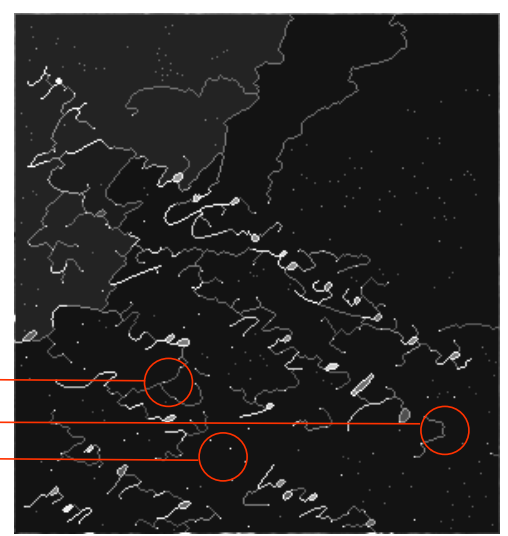

(Pink)

Figure 21: Results of skeletonization on the Persian letters manuscript. (DLD): $s=1.5$, low $=7$ and high $=21$. (SCCT): $\widehat{\sigma}_{\text {noise }}=7.93$ and $\gamma=10^{-5}$. (Pink): $\widehat{\lambda}=30$.

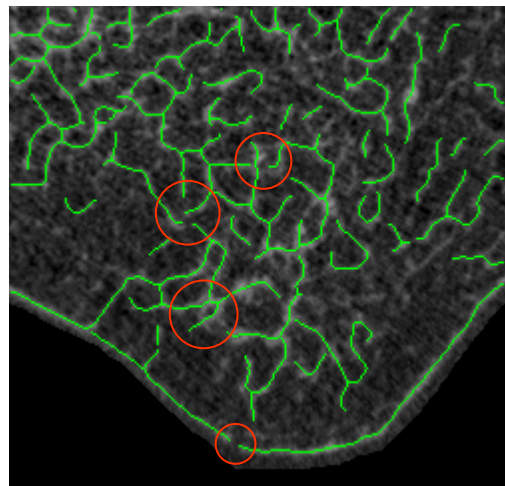

(DLD)

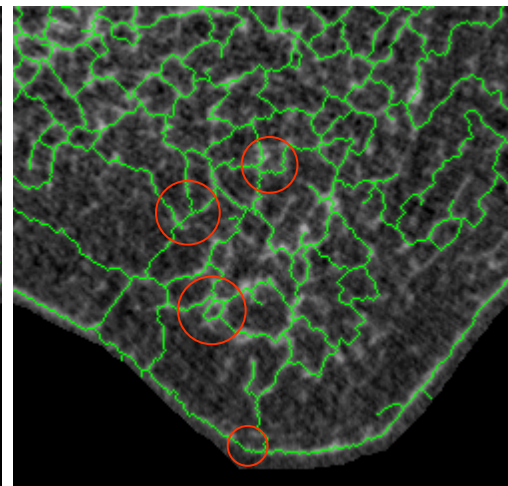

$(\mathrm{SCCT})$

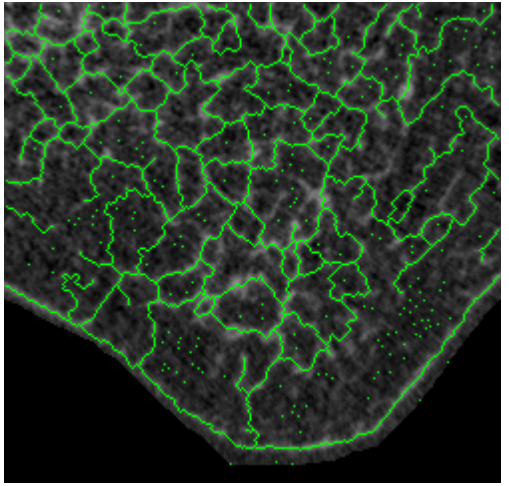

(Pink)

Figure 22: Skeletons superposed to initial image. (DLD): $s=2.5$, low $=0.2$ and upper threshold high $=0.8$. (SCCT): $\widehat{\sigma}_{\text {noise }}=4$ and $\gamma=5 \cdot 10^{-2}$. (Pink): $\widehat{\lambda}=\overline{\tau_{n}} \cdot \widehat{\sigma}_{\text {noise }}=16$.

According to the experimental results, one can point out the benefit of a local analysis leading to the determination of the parameter lambda for each examined pixel. At this stage, even though a spatial regularization strategy might sound appealing for the determination of a global parameter $\lambda$, it turns out that this parameter is statistically dependent on the local configuration $(\mu, \mathcal{K}, I(x)$ and $n)$. In this context, a first approach could be a spatial regularization to better estimate $\lambda$, which means enlarging the set of observations on which the mean of each dark connected component $(\mu)$ is estimated and thus, considering wider neighborhood. In addition to the fact that enlarging the neighborhood increases the algorithmic complexity of checking each enlarged dark component 
to estimate $\mu$, it's not obvious that such a regularization strategy could guarantee a good performance of the mean estimation. In fact, estimating $\mu$ on a wider dark neighborhood may introduce a bias in our calculus, since this strategy supposes a small variation of the mean, which is not the case in border regions. The strategy involving the nearest neighbors secures our estimation of $\mu$ and consequently the adjustment of $\lambda$.

A second spatial regularization strategy would aim at using a "global" parameter in certain regions of the image presenting, for example, the same background dynamic. The example of Figure 23 shows that such a strategy could impact the lowering of $\lambda$-crest pixels and by analogy, $\lambda$-peak and $\lambda$-end pixels.

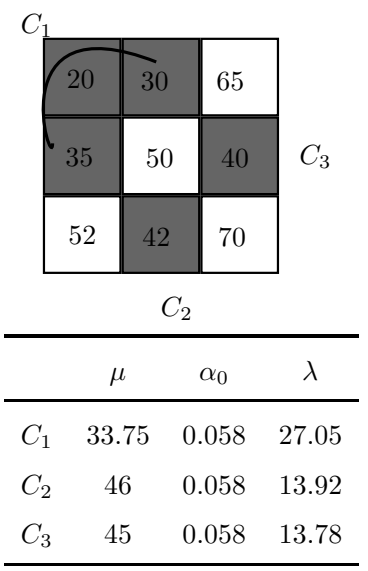

Case 1
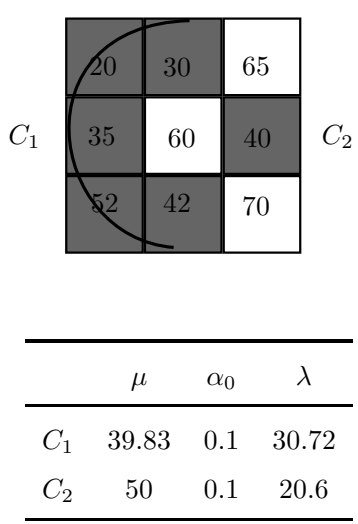

Case 2

Figure 23: Example of $\lambda_{k}$ calculus. Central pixel is $\lambda$-crest in both cases for $\alpha=0.01$ and $\sigma=5$.

For this example, we choose a global $\alpha=0.01$ and a noise standard deviation $\sigma=5$ to decide that central pixel is $\lambda$-crest. The difference between case 1 and case 2 is the gray value of the central pixel, while the background dynamic remains unchanged. By increasing the gray value of central pixel, we change not only the number of background components but also respective $\lambda_{k}$ values. In fact, even though component $C_{3}$ of case 1 and $C_{2}$ of case 2 are the same, the $\lambda_{k}$ associated is different in the two cases.

This configuration remains $\lambda$-lowerable in the two cases thanks to the new calculus of a local $\lambda_{k}$. Indeed, by choosing $\lambda_{3}$ of case 1 for testing $C_{2}$ in case2, this component could no longer be considered at a small distance from central pixel and thus, the lowering decision would not be feasible $\left(X_{(n)}-X_{(1)}=20>\lambda_{3}\right)$.

This example aims at showing the importance of the local control of $\lambda$, by taking into account not only the background information but also the gray level of the examined pixel. It also explains the difficulties encountered by $\lambda$-Skeleton method in some situations. 


\section{Conclusion}

We propose in this work a thinning method controlled by image self noise and contrast. It separates the adjustment of contrast and noise parameters through the use of the statistical framework of skeletonization useful in the analysis of bone micro-architecture. A switch of SCCT topological notions to the cubical complexes framework as used for the gray parallel thinning of Couprie et al. [24] would also be interesting.

\section{Acknowledgement}

This work was supported by ANR project Voxelo: ANR-12-TECS-0018. We would like to thank Professor Christine Chappard for providing project image. We also want to thank Professor Dhafer Maalouche for his useful comments and suggestions. 


\section{References}

[1] C. Steger, An unbiased detector of curvilinear structures., IEEE Trans. Pattern Anal. Mach. Intell. 20 (2) (1998) 113-125.

[2] B. S. Mayer, A. Baumgartner, C. Steger, H. Mayer, W. Eckstein, Multi-resolution, semantic objects, and context for road extraction, in: In Semantic Modeling for the Acquisition of Topographic Information from Images and Maps, Birkhauser Verlag, 1997, pp. 140-156.

[3] I. Laptev, H. Mayer, T. Lindeberg, W. Eckstein, C. Steger, A. Baumgartner, Automatic extraction of roads from aerial images based on scale space and snakes, Machine Vision and Applications 12 (1) (2000) 23-31.

[4] L. Lam, S.-W. Lee, C. Y. Suen, Thinning methodologies - a comprehensive survey, IEEE Trans. Pattern Anal. Mach. Intell. 14 (9) (1992) 869-885.

[5] B.-K. Jang, R. Chin, Analysis of thinning algorithms using mathematical morphology, Pattern Analysis and Machine Intelligence, IEEE Transactions on 12 (6) (1990) 541-551.

[6] B. Jang, R. Chin, One-pass parallel thinning: analysis, properties, and quantitative evaluation, IEEE Trans. Pattern Anal. Mach. Intell. 14 (11) (1992) 1129-1140.

[7] L. Lam, C. Suen, Evaluation of thinning algorithms from an ocr viewpoint, in: Document Analysis and Recognition, 1993., Proceedings of the Second International Conference on, 1993, pp. 287-290.

[8] Y.-S. Chen, Y.-T. YU, Thinning approach for noisy digital patterns, Pattern Recognition 29 (11) (1996) $1847-1862$.

[9] S. Wshah, Z. Shi, V. Govindaraju, Segmentation of arabic handwriting based on both contour and skeleton segmentation., in: ICDAR, IEEE Computer Society, 2009, pp. 793-797.

[10] J. Mena, J. Malpica, An automatic method for road extraction in rural and semi-urban areas starting from high resolution satellite imagery, Pattern Recognition Letters 26 (9) (2005) 1201 1220 .

[11] G. Lisini, C. Tison, F. Tupin, P. Gamba, Feature fusion to improve road network extraction in high-resolution sar images, Geoscience and Remote Sensing Letters, IEEE 3 (2) (2006) 217-221.

[12] P. Yim, P. Choyke, R. Summers, Gray-scale skeletonization of small vessels in magnetic resonance angiography, Medical Imaging, IEEE Transactions on 19 (6) (2000) 568-576.

[13] P. M. Patil, S. R. Suralkar, F. B. Sheikh, Rotation invariant thinning algorithm to detect ridge bifurcations for fingerprint identification., in: ICTAI, IEEE Computer Society, 2006, pp. 634-641. 
[14] F. Zhao, X. Tang, Preprocessing and postprocessing for skeleton-based fingerprint minutiae extraction, Pattern Recognition 40 (4) (2007) 1270 - 1281.

[15] M. Couprie, Note on fifteen 2d parallel thinning algorithms, Tech. rep., Institut Gaspard-Monge, Unit Mixte de Recherche CNRS-UMLV-ESIEE (2006).

[16] Rosenfeld, Connectivity in digital pictures, Journal of the ACM.

[17] V. Ranwez, P. Soille, Order independent homotopic thinning for binary and grey tone anchored skeletons, Pattern Recognition Letters 23 (6) (2002) 687-702.

${ }_{735}^{6}$ [18] M. Couprie, N. Bezerra, G. Bertrand, A parallel thinning algorithm for grayscale images, in: Discrete Geometry for Computer Imagery, Vol. 7749 of Lecture Notes in Computer Science, Springer Berlin Heidelberg, 2013, pp. 71-82.

[19] C. Arcelli, L. Serino, Skeletonization of labeled gray-tone images, Image and Vision Computing 23 (2) (2005) 159 - 166, discrete Geometry for Computer Imagery.

740 [20] J. Serra, Image Analysis and Mathematical Morphology, Academic Press, London, 1982.

[21] S. Beucher, J. Serra, Shapes and patterns of microstructures considered as grey-tone functions, in: 3rd European Symposium on Stereology, Ljubljana, 1981.

[22] G. Bertrand, J.-C. Everat, M. Couprie, Image segmentation through operators based on topology, Journal of Electronic Imaging 6 (4) (1997) 395-405.

[23] M. Couprie, F. Bezerra, G. Bertrand, Grayscale image processing using topological operators, in: SPIE Vision Geometry VIII., Vol. 3811, 1999, pp. 261-272.

[24] M. Couprie, N. Bezerra, G. Bertrand, A parallel thinning algorithm for grayscale images, in: Discrete Geometry for Computer Imagery, Vol. 7749 of Lecture Notes in Computer Science, Springer Berlin Heidelberg, 2013, pp. 71-82.

[25] G. Bertrand, On critical kernels, Comptes Rendus Mathematique 345 (7) (2007) 363 - 367.

[26] Y. Rabaa, S. Sevestre-Ghalila, A. Ricordeau, Contribution à la squelettisation en niveaux de gris, in: 42èmes Journées de Statistique, 2010.

[27] R. Youssef, S. Sevestre-Ghalilai, A. Ricordeau, Statistical control of thinning algorithm with implementation based on hierarchical queues, in: Soft Computing and Pattern Recognition (SoCPaR), 2014 6th International Conference of, 2014, pp. 365-370.

[28] P. Soille, Morphological Image Analysis, Springer-Verlag, 1999. 
[29] M. Cottrel, V. Genon-Catalot, G. RUGET, Exercices de probabilités avec rappel de cours, Dia, Belin, Paris, 1980, maîtrises de mathématiques pures et appliquées. Ecole d'ingénieurs.

[30] F. Benvenuto, A. La Camera, C. Theys, A. Ferrari, H. Lantri, M. Bertero, The study of an iterative method for the reconstruction of images corrupted by poisson and gaussian noise, Inverse Problems 24 (3) (2008) 035016.

[31] T. Nichols, Q. Jinyi, E. Asma, R. Leahy, Spatiotemporal reconstruction of list-mode pet data, Medical Imaging, IEEE Transactions on 21 (4) (2002) 396-404.

[32] P. Tseng, A modified forward-backward splitting method for maximal monotone mappings, SIAM Journal on Control and Optimization 38 (2) (2000) 431-446.

[33] M. Makitalo, A. Foi, Optimal inversion of the generalized anscombe transformation for poissongaussian noise, Image Processing, IEEE Transactions on 22 (1) (2013) 91-103.

[34] F. J. Anscombe, The Transformation of Poisson, Binomial, and Negative-Binomial Data, Biometrika 35 (3/4) (1948) 246-254.

[35] F. Meyer, Method for the processing of images by hierarchically organized queues, US Patent $5,463,698$ (oct 1995).

[36] R. Haralick, M. Jaisimha, D. Dori, A methodology for the characterization of the performance of thinning algorithms, in: Proceedings of the Second International Conference on Document Analysis and Recognition., 1993, pp. 282-286.

[37] M. Y. Jaisimha, R. Haralick, D. Dori, A methodology for the characterization of the performance of thinning algorithms, in: Proceedings of the Second International Conference on Document Analysis and Recognition., 1993, pp. 282-286.

[38] T. Grigorishin, G. H. Abdel-Hamid, Y.-H. Yang, Skeletonisation: An electrostatic field-based approach., Pattern Anal. Appl. 1 (3) (1998) 163-177.

[39] S. Lee, L. Lam, C. Suen, Performance evaluation of skeletonization algorithms for document analysis processing, in: Proceedings of the Second International Conference on Document Analysis and Recognition., 1991, pp. 260-271.

[40] J. Chaussard, M. Couprie, H. Talbot, Robust skeletonization using the discrete $\lambda$-medial axis, Pattern Recognition Letters 32 (9) (2011) 1384-1394.

[41] A. Al-Shatnawi, K. Omar, B. AlFawwaz, A. Zeki, Skeleton extraction: Comparison of five methods on the arabic ifn/enit database, in: Computer Science and Information Technology (CSIT), 2014 6th International Conference on, 2014, pp. 50-59. 
[42] C. Heipke, H. Mayer, C. Wiedemann, O. Jamet, Evaluation of automatic road extraction, in: In: International Archives of Photogrammetry and Remote Sensing, 1997, pp. 47-56.

[47] S. Mozaffari, K. Faez, M. Ziaratban, Structural decomposition and statistical description of farsi/arabic handwritten numeric characters, in: Document Analysis and Recognition, 2005. Proceedings. Eighth International Conference on, 2005, pp. 237-241 Vol. 1.

[48] L. Lorigo, V. Govindaraju, Offline arabic handwriting recognition: a survey, Pattern Analysis and

[49] S. Wshah, Z. Shi, V. Govindaraju, Segmentation of arabic handwriting based on both contour and skeleton segmentation, in: Document Analysis and Recognition, 2009. ICDAR '09. 10th International Conference on, 2009, pp. 793-797.

[50] A. Laib, P. Regsegger, Calibration of trabecular bone structure measurements of in vivo threedimensional peripheral quantitative computed tomography with $28-\mu \mathrm{m}$-resolution microcomputed tomography, Bone 24 (1) (1999) 35 - 39.

[51] S. Sevestre-Ghalila, A. Benazza-Benyahia, A. Ricordeau, N. Mellouli, C. Chappard, C. Benhamou, Texture image analysis for osteoporosis detection with morphological tools, in: Medical Image Computing and Computer-Assisted Intervention MICCAI 2004, Vol. 3216 of Lecture Notes in Computer Science, Springer Berlin Heidelberg, 2004, pp. 87-94.

[52] A. Almhdie, O. Rozenbaum, E. Lespessailles, R. Jennane, Image processing for the non-destructive characterization of porous media. Application to limestones and trabecular bones, Mathematics and Computers in Simulation 99 (2014) 82-94. 


\section{Vitae}

About the author- Rabaa Youssef graduated from the National School of Computer Science (ENSI, Tunisia) in 2006 and worked as an engineer in STMicroelectronics for 2 years. She received her Master of Science degree in applied mathematics from the University of Paris Descartes and the University of El Manar in 2009. She received a Ph.D degree in november 2015 under a joint graduation between the University of Carthage, Tunisa (ICT doctorate) and the University of Pierre et Marie Curie

${ }_{825}$ (Applied mathematics doctorate). Her current researches include mathematical morphology tools for biomedical imaging and is actually involved in Voxelo project.

About the author- Sylvie Sevestre-Ghalila earned her Ph.D. from the University of Paris Sud, Centre d'Orsay, France, in 1993. From 1994 up to now, she is an Assistant Professor at the Mathematics and Computer Sciences Dpt and, France, member of the laboratory Applied Mathematics of Paris Descartes and member of the French Society of Statistic (SFdS). She is actually head of the CEALinklab, a joint laboratory between the Technology Research Division of the Atomic Energy and Alternative Energies Commission (DRT-CEA, France) and Telnet Innovation Labs company (Tunisia). Her research interests are Statistic, Markov random field, stochastic image processing, medical imaging, biomedical imaging and visualization.

About the author- Anne Ricordeau earned her Ph.D. from the University of Paris Sud, 745 Centre d'Orsay, France, in 1993. She is currently Assistant Professor at the University Institute of Technology of Montreuil, France, member of the applied mathematics laboratory (MAP5) of Paris Descartes University. Her research interests are Statistic, stochastic image processing and biomedical imaging and visualization.

About the author- Amel Benazza-Benyahia received the engineering degree from the National Institute of Telecommunications, Evry, France, in 1988, the Ph.D. degree from the Universit ParisSud (XI), Paris, France, in 1993, and the Habilitation Universitaire from the Ecole Supérieure des Communications (SUP'COM), Tunis, Tunisia, in 2003. She is currently a full Professor with the Department of Applied Mathematics, Signal Processing and Communications, SUP'COM. She is also a Research Scientist with the Communication, Signal and Image Lab. (COSIM). Her research interests include multispectral image compression, signal denoising, and medical image analysis. 